\title{
Optimal Control of Non-Smooth Hyperbolic Evolution Maxwell Equations in
} Type-II Superconductivity

\author{
Yousept, Irwin
}

This text is provided by DuEPublico, the central repository of the University Duisburg-Essen.

This version of the e-publication may differ from a potential published print or online version.

DOI: https://doi.org/10.1137/16M1074229

URN: urn:nbn:de:hbz:464-20180829-110303-0

Link: https://duepublico.uni-duisburg-essen.de:443/servlets/DocumentServlet?id=46801

Legal notice:

(C) 2017 Society for Industrial and Applied Mathematics

Source: First Published in SIAM J. Control Optim. in Vol. 55, No.4, published by the Society for Industrial and Applied Mathematics (SIAM). 


\title{
OPTIMAL CONTROL OF NON-SMOOTH HYPERBOLIC EVOLUTION MAXWELL EQUATIONS IN TYPE-II SUPERCONDUCTIVITY*
}

\author{
IRWIN YOUSEPT ${ }^{\dagger}$
}

\begin{abstract}
We analyze the optimal control of an electromagnetic process in type-II superconductivity. The PDE-constrained optimization problem is to find an optimal applied current density, which steers the electromagnetic fields to the desired ones in the presence of a type-II superconductor. The governing PDE system for the electromagnetic fields consists of hyperbolic evolution Maxwell equations with a nonlinear and nonsmooth constitutive law for the electric field and the current density based on the Bean critical-state model. Through the use of the Maxwell theory, the semigroup theory, Helmholtz decomposition, and results on maximal monotone operators, we develop a mathematical theory including an existence analysis and first-order necessary optimality conditions for the nonsmooth PDE-constrained optimization problem.
\end{abstract}

Key words. nonsmooth hyperbolic evolution Maxwell equations, Bean's critical-state model, type-II superconductivity, nonsmooth PDE-constrained optimization, existence analysis, optimality system

AMS subject classifications. 35Q60, 35Q61, 35Q93

DOI. $10.1137 / 16 \mathrm{M} 1074229$

1. Introduction. If a superconductor is cooled down below its critical temperature $T_{c}$, then it looses its electrical resistivity. This is the fundamental nature of superconductivity, which was discovered in 1911 by Onnes. Based on this property, superconductors can transfer an electric current without energy dissipation. The second underlying property of superconductivity is the Meissner effect: If an external weak magnetic field is applied to a superconductor at a temperature below its critical temperature $T_{c}$, then the magnetic flux is completely expelled from the superconductor (Figure 1). Today, superconductivity makes many new applications and key technologies possible, including magnetic resonance imaging, magnetic confinement fusion technologies, high-energy particle accelerators, magnetic levitation technologies, magnetic energy storage, and many more.

Superconductors are classified into type-I and type-II. The first type is characterized as follows. The Meissner effect occurs under the condition that the applied magnetic field strength is below a certain critical level $H_{c}$. Above this threshold, the superconducting state suddenly breaks down (sharp transition to the normal state) and the magnetic flux penetrates into the material. Type-I superconductors are mostly pure metals (mercury, aluminium, gallium, etc.) and admit extremely low critical temperatures. Furthermore, the superconducting state can already be destroyed by applying a not so strong magnetic field. For this reason, the application of type-I superconductors is rather limited. The physical behavior of the second type is distinctly different from the first one (see Figure 2). It features two critical values of magnetic field $H_{c 1}<H_{c 2}$. As long as the magnetic field strength is below the lower critical

*Received by the editors May 6, 2016; accepted for publication (in revised form) March 8, 2017; published electronically July 27, 2017.

http://www.siam.org/journals/sicon/55-4/M107422.html

Funding: This work was supported by the German Research Foundation Priority Program DFG SPP 1962 Project YO 159/2-1.

${ }^{\dagger}$ Fakultät für Mathematik, Universität Duisburg-Essen, Thea-Leymann-Str. 9, D-45127 Essen, Germany (irwin.yousept@uni-due.de). 


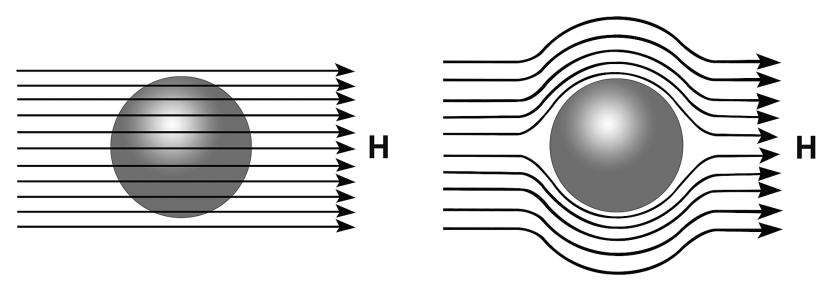

FIG. 1. Normal state of a superconductor at a temperature above $T_{c}$ (left plot) and the Meissner effect in a superconductor at a temperature below $T_{c}$ (right plot).

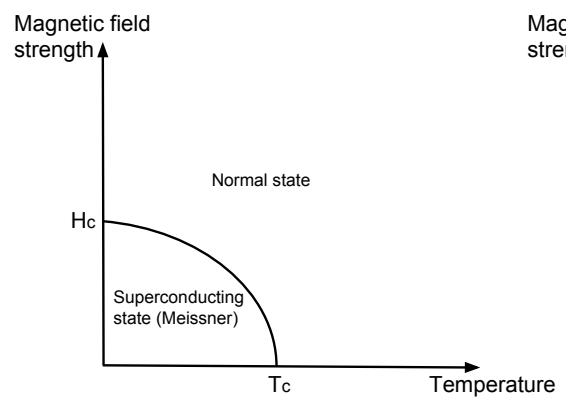

Type-I superconductivity

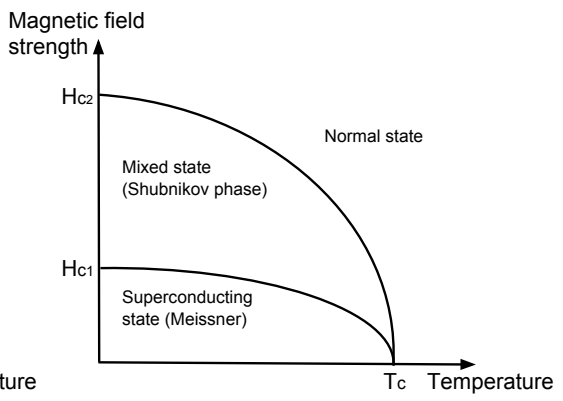

Type- II superconductivity

FIG. 2. Sharp transition to the normal state in typ-I superconductors (left plot) and the mixed state in type-II superconductors (right plot).

value $H_{c 1}$, the magnetic flux is excluded from the superconductor (Meissner effect). Once the magnetic field strength is greater than $H_{c 1}$ and less than the upper critical value $H_{c_{2}}$, then the magnetic flux penetrates partially into the material, but the superconducting state is not fully destroyed. This kind of physical state is called the Shubnikov phase or mixed state. Finally, the superconducting state completely breaks down if the magnetic field strength is increased above the upper critical level $H_{c 2}$. At this stage, the magnetic flux passes through the material completely. Type-II superconductors admit higher critical temperatures (high-temperature superconductivity) and greater critical values of magnetic field than those of the first kind. These properties enable them to preserve their superconducting effects in the presence of a strong applied magnetic field at higher temperatures. Today, most technological applications of superconductors are based on the use of the second type.

The Shubnikov phase is the key feature of type-II superconductivity. Being in the mixed state, a superconductor of type-II allows partial penetration of the magnetic flux in the form of flux tubes. Each of these tubes carries a single magnetic flux quantum and is surrounded by a supercurrent vortex. A change in the applied magnetic field leads to a change in the density of the flux tubes and the supercurrent vortices. However, the dynamic process in response to the time-varying magnetic field is not reversible and exhibits hysteresis. Based on experimental observations, Bean $[6,7]$ proposed a critical-state model which describes the irreversible magnetization process in type-II superconductivity. The model postulates a constitutive relation between the electric field and the current density as follows:

(A1) the current density strength cannot exceed some critical value $\mathfrak{j}_{c}$;

(A2) the electric field vanishes if the current density strength is strictly less than $\mathfrak{j}_{c}$;

(A3) the electric field is parallel to the current density. 
Suppose that $\Omega \subset \mathbb{R}^{3}$ is a bounded Lipschitz domain filled with isotropic materials. Inside this medium, there is a domain $\Omega_{s c}$, satisfying $\bar{\Omega}_{s c} \subset \Omega$, which represents a type-II superconductor. The dynamic of the electromagnetic fields in $\Omega$ is governed by the Maxwell equations consisting of first-order hyperbolic partial differential equations:

$$
\begin{cases}\epsilon \boldsymbol{E}_{t}-\operatorname{curl} \boldsymbol{H}+\boldsymbol{J}=\boldsymbol{u} & \text { in } \Omega \times(0, T), \\ \mu \boldsymbol{H}_{t}+\operatorname{curl} \boldsymbol{E}=0 & \text { in } \Omega \times(0, T), \\ \boldsymbol{E} \times \boldsymbol{n}=0 & \text { on } \partial \Omega \times(0, T), \\ \boldsymbol{E}(\cdot, 0)=\boldsymbol{E}_{0} & \text { in } \Omega, \\ \boldsymbol{H}(\cdot, 0)=\boldsymbol{H}_{0} & \text { in } \Omega .\end{cases}
$$

Here, $\boldsymbol{E}: \Omega \times(0, T) \rightarrow \mathbb{R}^{3}$ denotes the electric field, $\boldsymbol{H}: \Omega \times(0, T) \rightarrow \mathbb{R}^{3}$ the magnetic field, $\boldsymbol{J}: \Omega \times(0, T) \rightarrow \mathbb{R}^{3}$ the current density, and $\boldsymbol{E}_{0}, \boldsymbol{H}_{0}: \Omega \rightarrow \mathbb{R}^{3}$ the initial electric and magnetic fields. Furthermore, the functions $\epsilon, \mu: \Omega \rightarrow \mathbb{R}$ stand for the electric permittivity and the magnetic permeability, respectively. In the right-hand side of the Maxwell-Ampère equation, the vector field $\boldsymbol{u}: \Omega \times(0, T) \rightarrow \mathbb{R}^{3}$ represents the applied current source. As boundary condition, we employ the standard perfectly conducting electric boundary condition, where $\boldsymbol{n}$ denotes the unit outward normal to $\partial \Omega$.

During the electromagnetic process, the temperature of the superconductor $\Omega_{s c}$ is assumed to be constant and to stay below its critical temperature. This gives rise to the superconducting state, as described above. Outside the superconductor $\Omega_{s c}$, we suppose that the current density $\boldsymbol{J}$ vanishes. Based on this physical assumption, a nonlinear and nonsmooth relation for $\boldsymbol{E}$ and $\boldsymbol{J}$ is obtained as follows (see (A1)-(A3)):

$$
\begin{cases}\boldsymbol{J}(x, t) \cdot \boldsymbol{E}(x, t)=j_{c}(x)|\boldsymbol{E}(x, t)| & \text { for a.e. }(x, t) \in \Omega \times(0, T), \\ |\boldsymbol{J}(x, t)| \leq j_{c}(x) & \text { for a.e. }(x, t) \in \Omega \times(0, T) .\end{cases}
$$

Here, the function $j_{c}: \Omega \rightarrow \mathbb{R}$ is given by

$$
j_{c}:= \begin{cases}\mathfrak{j}_{c} & \text { in } \Omega_{s c} \\ 0 & \text { in } \Omega \backslash \bar{\Omega}_{s c},\end{cases}
$$

where $\mathfrak{j}_{c} \in \mathbb{R}^{+}$denotes the critical current density of $\Omega_{s c}$ as postulated in (A1). We note that $(1.1 \mathrm{~b})$ is equivalent to the following conditions:

$$
\boldsymbol{J}(x, t)=j_{c}(x) \frac{\boldsymbol{E}(x, t)}{|\boldsymbol{E}(x, t)|} \text {, if } \boldsymbol{E}(x, t) \neq 0, \quad \text { and } \quad|\boldsymbol{J}(x, t)| \leq j_{c}(x) \text {, if } \boldsymbol{E}(x, t)=0,
$$

for a.e. $(x, t) \in \Omega \times(0, T)$.

If the displacement current $\epsilon \boldsymbol{E}_{t}$ is significantly smaller compared with $-\operatorname{curl} \boldsymbol{H}+$ $\boldsymbol{J}$, then the Maxwell equations (1.1a) can be approximated by neglecting $\epsilon \boldsymbol{E}_{t}$. This approximation is called eddy current approximation (see [1]), which simplifies the non-smooth hyperbolic Maxwell system (1.1) to a magnetic field formulation in form of a parabolic variational inequality. Prigozhin $[18,17]$ was the first, who introduced and analyzed this $\boldsymbol{H}$-formulation. Furthermore, the finite element analysis for the associated three-dimensional (3D) parabolic variational inequality was investigated in [9]. Earlier mathematical results on a 2D parabolic p-Laplacian problem arising in type-II superconductivity with a nonlinear critical current state $j_{c}$, which leads to a quasi-variational inequality problem, can be found in [19] (see also [5]). All the aforementioned contributions are devoted to the (parabolic) eddy current approximation of the nonsmooth hyperbolic Maxwell system (1.1). The analysis of the Maxwell system (1.1) in the presence of the displacement current $\epsilon \boldsymbol{E}_{t}$ goes back to Jochmann [13, 14]. 
1.1. Nonsmooth PDE-constrained optimization. This paper addresses the mathematical analysis for an optimal control problem of the electromagnetic process (1.1). More precisely, we look for an optimal current source $\boldsymbol{u}: \Omega \times(0, T) \rightarrow \mathbb{R}^{3}$, which steers the time-dependent electromagnetic fields $\boldsymbol{E}$ and $\boldsymbol{H}$ toward the desired ones in the presence of the type-II superconductor $\Omega_{s c}$. This leads to the following PDE-constrained optimization problem:

$$
\begin{aligned}
\min \mathcal{J}(\boldsymbol{E}, \boldsymbol{H}, \boldsymbol{u}):= & \frac{1}{2}\left\|\boldsymbol{E}-\boldsymbol{E}_{d}\right\|_{L^{2}\left((0, T), \boldsymbol{L}^{2}(\Omega)\right)}^{2}+\frac{1}{2}\left\|\boldsymbol{H}-\boldsymbol{H}_{d}\right\|_{L^{2}\left((0, T), \boldsymbol{L}^{2}(\Omega)\right)}^{2} \\
& +\frac{\kappa}{2}\|\boldsymbol{u}\|_{H^{2}\left((0, T), \boldsymbol{L}^{2}(\Omega)\right)}^{2},
\end{aligned}
$$

subject to the nonsmooth hyperbolic evolution Maxwell system

$$
\begin{cases}\epsilon \boldsymbol{E}_{t}-\operatorname{curl} \boldsymbol{H}+\boldsymbol{J}=\boldsymbol{u} & \text { in } \Omega \times(0, T), \\ \mu \boldsymbol{H}_{t}+\operatorname{curl} \boldsymbol{E}=0 & \text { in } \Omega \times(0, T), \\ \boldsymbol{E} \times \boldsymbol{n}=0 & \text { on } \partial \Omega \times(0, T), \\ \boldsymbol{E}(\cdot, 0)=\boldsymbol{E}_{0} & \text { in } \Omega, \\ \boldsymbol{H}(\cdot, 0)=\boldsymbol{H}_{0} & \text { in } \Omega, \\ \boldsymbol{J}(x, t) \cdot \boldsymbol{E}(x, t)=j_{c}(x)|\boldsymbol{E}(x, t)| & \text { a.e. in } \Omega \times(0, T), \\ |\boldsymbol{J}(x, t)| \leq j_{c}(x) & \text { a.e. in } \Omega \times(0, T),\end{cases}
$$

and to the divergence-free constraint on the control

$$
\operatorname{div} \boldsymbol{u}=0 \quad \text { in } \Omega \times(0, T) .
$$

In the setting of $(\mathrm{P}), \kappa>0$ is the control cost term and $\boldsymbol{E}_{d}, \boldsymbol{H}_{d}: \Omega \times(0, T) \rightarrow \mathbb{R}^{3}$ are the desired electromagnetic fields. Furthermore, we include the divergence-free control constraint (1.3) on the applied current source. This condition arises from the physical charge conservation law. In addition to the divergence-free condition, the PDE-constrained optimization problem $(\mathrm{P})$ also considers controls with a higher time-regularity property. This regularity is mainly required in order to obtain the strong solution of the Maxwell system (1.2), which turns out to be crucial for our analysis.

To the best of the author's knowledge, this article is the first study on a PDEconstrained optimization problem governed by nonsmooth and nonlinear evolution Maxwell equations. Almost all studies on the optimal control of Maxwell's equations were devoted to the linear case $[8,11,22,15,23,24]$. So far, the nonlinear case [25] has only been investigated under a stationary (magnetostatic) and smooth assumption. The contribution of the present paper is the mathematical analysis for $(\mathrm{P})$, including an existence analysis and first-order necessary optimality conditions, where the key tools for the analysis are the theory of Maxwell's equations, the semigroup theory, results on maximal monotone operators, Helmholtz decomposition, and the penalization technique by Barbu [4] (cf. [12]).

2. Preliminaries. We begin by introducing our notation and the mathematical assumption on the data involved in (1.2) and (P). Throughout this paper, $c$ denotes a generic positive constant that can take different values on different occasions. For a given Hilbert space $V$, we use the notation $\|\cdot\|_{V}$ and $(\cdot, \cdot)_{V}$ for a standard norm and a standard scalar product in $V$. By $V^{*}$, we denote the dual space of $V$, and, for the associated duality pairing, we write $\langle\cdot, \cdot\rangle_{V^{*}, V}$. Furthermore, if $V$ is continuously embedded in another normed linear space $Y$, we write $V \hookrightarrow Y$. By $\mathcal{L}(X, Y)$, we 
denote the space of all linear and bounded operators between normed linear spaces $X$ and $Y$. We use a bold typeface to indicate a $3 \mathrm{D}$ vector function or a Hilbert space of $3 \mathrm{D}$ vector functions. The main Hilbert spaces we use in our analysis are

$$
\begin{aligned}
\boldsymbol{H}(\text { curl }) & :=\left\{\boldsymbol{q} \in \boldsymbol{L}^{2}(\Omega) \mid \operatorname{curl} \boldsymbol{q} \in \boldsymbol{L}^{2}(\Omega),\right. \\
\boldsymbol{H}_{0}(\text { curl }) & :=\{\boldsymbol{q} \in \boldsymbol{H}(\operatorname{curl}) \mid \boldsymbol{q} \times \boldsymbol{n}=0 \text { on } \partial \Omega, \\
\boldsymbol{H}(\operatorname{div}) & :=\left\{\boldsymbol{q} \in \boldsymbol{L}^{2}(\Omega) \mid \operatorname{div} \boldsymbol{q} \in L^{2}(\Omega),\right. \\
\boldsymbol{H}(\operatorname{div}=0) & :=\left\{\boldsymbol{q} \in \boldsymbol{L}^{2}(\Omega) \mid(\boldsymbol{q}, \nabla \phi)_{\boldsymbol{L}^{2}(\Omega)}=0 \quad \forall \phi \in H_{0}^{1}(\Omega),\right.
\end{aligned}
$$

where the curl- and div-operators as well as the tangential trace are understood in the sense of distributions. We also introduce the weighted Hilbert space

$$
\boldsymbol{X}:=\boldsymbol{L}_{\epsilon}^{2}(\Omega) \times \boldsymbol{L}_{\mu}^{2}(\Omega),
$$

equipped with the (weighted) scalar product

$$
((\boldsymbol{y}, \boldsymbol{w}),(\widehat{\boldsymbol{y}}, \widehat{\boldsymbol{w}}))_{\boldsymbol{X}}=(\epsilon \boldsymbol{y}, \widehat{\boldsymbol{y}})_{L^{2}(\Omega)}+(\mu \boldsymbol{w}, \widehat{\boldsymbol{w}})_{L^{2}(\Omega)} \quad \forall(\boldsymbol{y}, \boldsymbol{w}),(\widehat{\boldsymbol{y}}, \widehat{\boldsymbol{w}}) \in \boldsymbol{X}
$$

Let us now define the Maxwell operator

$$
\mathcal{A}: D(\mathcal{A}) \subset \boldsymbol{X} \rightarrow \boldsymbol{X}, \quad \mathcal{A}:=-\left(\begin{array}{cc}
0 & -\epsilon^{-1} \text { curl } \\
\mu^{-1} \text { curl } & 0
\end{array}\right),
$$

where the domain of $\mathcal{A}$ is given by

$$
D(\mathcal{A}):=\boldsymbol{H}_{0}(\text { curl }) \times \boldsymbol{H}(\operatorname{curl}) .
$$

Assumption 2.1. The electric permittivity $\epsilon: \Omega \rightarrow \mathbb{R}$ and the magnetic permeability $\mu: \Omega \rightarrow \mathbb{R}$ are assumed to be Lebesgue measurable and essentially bounded. There exist positive constants $0<\underline{\epsilon}<\bar{\epsilon}<\infty$ and $0<\underline{\mu}<\bar{\mu}<\infty$ such that

$$
\underline{\epsilon} \leq \epsilon(x) \leq \bar{\epsilon} \quad \text { a.e. in } \Omega \quad \text { and } \quad \underline{\mu} \leq \mu(x) \leq \bar{\mu} \quad \text { a.e. in } \Omega .
$$

The initial data $\boldsymbol{E}_{0}, \boldsymbol{H}_{0}$ and the desired electromagnetic fields $\boldsymbol{E}_{d}, \boldsymbol{H}_{d}$ are assumed to satisfy $\left(\boldsymbol{E}_{0}, \boldsymbol{H}_{0}\right) \in D(\mathcal{A})$ and $\left(\boldsymbol{E}_{d}, \boldsymbol{H}_{d}\right) \in L^{2}((0, T), \boldsymbol{X})$.

2.1. Well-known results. Employing the Maxwell operator $\mathcal{A}$, the evolution Maxwell equations (1.1a) can be equivalently formulated as the following Cauchy problem:

$$
\left\{\begin{aligned}
\left(\frac{d}{d t}-\mathcal{A}\right)(\boldsymbol{E}, \boldsymbol{H})(t) & =\left(\epsilon^{-1}(\boldsymbol{u}(t)-\boldsymbol{J}(t)), 0\right), \quad t \in(0, T), \\
(\boldsymbol{E}, \boldsymbol{H})(0) & =\left(\boldsymbol{E}_{0}, \boldsymbol{H}_{0}\right) .
\end{aligned}\right.
$$

We note that the operator $\mathcal{A}: D(\mathcal{A}) \subset \boldsymbol{X} \rightarrow \boldsymbol{X}$ is skew-adjoint, i.e., it holds for the corresponding adjoint operator that $\mathcal{A}^{*}=-\mathcal{A}$ and $D\left(\mathcal{A}^{*}\right)=D(\mathcal{A})=\boldsymbol{H}_{0}(\mathbf{c u r l}) \times$ $\boldsymbol{H}$ (curl). Therefore, by virtue of Stone's theorem [10, Theorem 3.24, p. 89], the operator $\mathcal{A}$ generates a strongly continuous group $\left\{\mathbb{T}_{t}\right\}_{t \in \mathbb{R}}$ of unitary operators on $\boldsymbol{X}$.

Copyright (C) by SIAM. Unauthorized reproduction of this article is prohibited. 
Definition 2.2. Let $\boldsymbol{u} \in L^{2}\left((0, T), \boldsymbol{L}^{2}(\Omega)\right)$. A triple $(\boldsymbol{E}, \boldsymbol{H}, \boldsymbol{J}) \in \mathcal{C}([0, T], \boldsymbol{X}) \times$ $L^{2}\left((0, T), \boldsymbol{L}^{2}(\Omega)\right)$ is called a mild solution of (1.2) associated with $\boldsymbol{u}$ if and only if

$$
\left\{\begin{array}{l}
(\boldsymbol{E}, \boldsymbol{H})(t)=\mathbb{T}_{t}\left(\boldsymbol{E}_{0}, \boldsymbol{H}_{0}\right)+\int_{0}^{t} \mathbb{T}_{t-s}\left(\epsilon^{-1}(\boldsymbol{u}(s)-\boldsymbol{J}(s)), 0\right) d s \quad \forall t \in[0, T], \\
\boldsymbol{J}(x, t) \cdot \boldsymbol{E}(x, t)=j_{c}(x)|\boldsymbol{E}(x, t)| \text { for a.e. }(x, t) \in \Omega \times(0, T), \\
|\boldsymbol{J}(x, t)| \leq j_{c}(x) \quad \text { for a.e. }(x, t) \in \Omega \times(0, T) .
\end{array}\right.
$$

The existence of a unique mild solution to (1.2) has been proven by Jochmann in [13, Theorem 1]. We summarize the corresponding existence and uniqueness result in the following lemma.

Lemma 2.3. For every $\boldsymbol{u} \in L^{2}\left((0, T), \boldsymbol{L}^{2}(\Omega)\right)$, the Maxwell system (1.2) admits a unique mild solution $(\boldsymbol{E}, \boldsymbol{H}, \boldsymbol{J}) \in \mathcal{C}([0, T], \boldsymbol{X}) \times L^{2}\left((0, T), \boldsymbol{L}^{2}(\Omega)\right)$.

If $\boldsymbol{u} \in W^{1, \infty}\left((0, T), \boldsymbol{L}^{2}(\Omega)\right)$, then the mild solution turns out to be the strong solution of (1.2). This result has been justified in [14, Lemma 4.3].

Lemma 2.4. For every $\boldsymbol{u} \in W^{1, \infty}\left((0, T), \boldsymbol{L}^{2}(\Omega)\right)$, the mild solution $(\boldsymbol{E}, \boldsymbol{H}, \boldsymbol{J}) \in$ $\mathcal{C}([0, T], \boldsymbol{X}) \times L^{2}\left((0, T), \boldsymbol{L}^{2}(\Omega)\right)$ of (1.2) enjoys the regularity property

$$
(\boldsymbol{E}, \boldsymbol{H}) \in L^{\infty}((0, T), D(\mathcal{A})) \cap W^{1, \infty}((0, T), \boldsymbol{X})
$$

and satisfies (2.2) for almost all $t \in(0, T)$, i.e., it is the (unique) strong solution of the Maxwell system (1.2) associated with $\boldsymbol{u}$. Furthermore, it holds that

$$
\|(\boldsymbol{E}, \boldsymbol{H})\|_{L^{\infty}((0, T), D(\mathcal{A})) \cap W^{1, \infty}((0, T), \boldsymbol{X})} \leq c\left(\|\boldsymbol{u}\|_{W^{1, \infty}\left((0, T), \boldsymbol{L}^{2}(\Omega)\right)}+1\right)
$$

with a constant $c>0$, independent of $\boldsymbol{u}$ and $(\boldsymbol{E}, \boldsymbol{H}, \boldsymbol{J})$.

Remark 2.5. Thanks to the injection $H^{2}\left((0, T), \boldsymbol{L}^{2}(\Omega)\right) \hookrightarrow W^{1, \infty}\left((0, T), \boldsymbol{L}^{2}(\Omega)\right)$, the strong regularity result of Lemma 2.4 is satisfied for every feasible control $\boldsymbol{u} \in$ $H^{2}((0, T), \boldsymbol{H}(\operatorname{div}=0))$ of $(\mathrm{P})$.

We close this section by recalling a classical result on the energy balance equality for every strongly continuous group of unitary operators on $\boldsymbol{X}$.

Lemma 2.6. Let $\left\{\mathbb{S}_{t}\right\}_{t \in \mathbb{R}}$ be a strongly continuous group of unitary operators on $\boldsymbol{X}$. Furthermore, suppose that $(\boldsymbol{e}, \boldsymbol{h}) \in \mathcal{C}([0, T], \boldsymbol{X}),\left(\boldsymbol{e}_{0}, \boldsymbol{h}_{0}\right) \in \boldsymbol{X}$, and $(\boldsymbol{w}, \hat{\boldsymbol{w}}) \in$ $L^{1}((0, T), \boldsymbol{X})$ satisfy

$$
(\boldsymbol{e}, \boldsymbol{h})(t)=\mathbb{S}_{t}\left(\boldsymbol{e}_{0}, \boldsymbol{h}_{0}\right)+\int_{0}^{t} \mathbb{S}_{t-s}(\boldsymbol{w}, \hat{\boldsymbol{w}})(s) d s \quad \forall t \in[0, T] .
$$

Then, the energy balance equality

$$
\|(\boldsymbol{e}, \boldsymbol{h})(t)\|_{\boldsymbol{X}}^{2}=\left\|\left(\boldsymbol{e}_{0}, \boldsymbol{h}_{0}\right)\right\|_{\boldsymbol{X}}^{2}+2 \int_{0}^{t}((\boldsymbol{w}, \hat{\boldsymbol{w}})(s),(\boldsymbol{e}, \boldsymbol{h})(s))_{\boldsymbol{X}} d s
$$

holds for all $t \in[0, T]$.

3. Existence analysis. The existence analysis of $(\mathrm{P})$ is mainly complicated by the fact that the injections

$$
\boldsymbol{H}(\operatorname{div}=0) \hookrightarrow \boldsymbol{L}^{2}(\Omega) \quad \text { and } \quad \boldsymbol{H}_{0}(\operatorname{curl}) \hookrightarrow \boldsymbol{L}^{2}(\Omega)
$$

Copyright (C) by SIAM. Unauthorized reproduction of this article is prohibited. 
are not compact (see, for instance, [2, Proposition 2.7]). Thus, classical arguments based on the compactness of the injection of the state space (or the control space) to the Hilbert space $L^{2}\left((0, T), \boldsymbol{L}^{2}(\Omega)\right)$ cannot be used for proving the existence. Here, our main idea is to make use of the analytical properties from the nonsmooth relation (1.1b), the divergence-free constraint (1.3), and the regularity result for the electromagnetic fields (Lemma 2.4) in combination with the Helmholtz decomposition:

$$
\boldsymbol{L}^{2}(\Omega)=\nabla H_{0}^{1}(\Omega) \oplus \boldsymbol{H}(\operatorname{div}=0) .
$$

According to (3.1), for every $\mathbf{F} \in \boldsymbol{L}^{2}(\Omega)$, there exists a unique pair $(\phi, \widehat{\mathbf{F}}) \in H_{0}^{1}(\Omega) \times$ $\boldsymbol{H}(\operatorname{div}=0)$ such that

$$
\mathbf{F}=\nabla \phi+\widehat{\mathbf{F}}
$$

We denote the associated Helmholtz projection by $\boldsymbol{\pi}: \boldsymbol{L}^{2}(\Omega) \rightarrow \boldsymbol{H}(\operatorname{div}=0), \mathbf{F} \rightarrow \widehat{\mathbf{F}}$. By definition, $\boldsymbol{\pi}: \boldsymbol{L}^{2}(\Omega) \rightarrow \boldsymbol{H}(\operatorname{div}=0)$ is a linear and bounded operator satisfying

$$
(\boldsymbol{v}, \boldsymbol{\pi} \mathbf{F})_{\boldsymbol{L}^{2}(\Omega)}=(\boldsymbol{v}, \mathbf{F})_{\boldsymbol{L}^{2}(\Omega)} \quad \forall \boldsymbol{v} \in \boldsymbol{H}(\operatorname{div}=0), \quad \forall \mathbf{F} \in \boldsymbol{L}^{2}(\Omega) .
$$

Furthermore, since $\nabla H_{0}^{1}(\Omega) \subset \boldsymbol{H}_{0}(\mathbf{c u r l})$ and $\operatorname{curl} \nabla \equiv 0$, the Helmholtz projection $\boldsymbol{\pi}$ considered as an operator from $\boldsymbol{H}_{0}(\mathbf{c u r l})$ to $\boldsymbol{H}_{0}(\mathbf{c u r l}) \cap \boldsymbol{H}(\operatorname{div}=0)$ is also linear and bounded. In conclusion,

$$
\boldsymbol{\pi} \in \mathcal{L}\left(\boldsymbol{L}^{2}(\Omega), \boldsymbol{H}(\operatorname{div}=0)\right) \quad \text { and } \quad \boldsymbol{\pi} \in \mathcal{L}\left(\boldsymbol{H}_{0}(\operatorname{curl}), \boldsymbol{H}_{0}(\operatorname{curl}) \cap \boldsymbol{H}(\operatorname{div}=0)\right) .
$$

As a consequence of (3.3), we obtain the following result.

COROLlary 3.1. The Helmholtz projection $\boldsymbol{\pi}$ is linear and bounded considered as an operator from the Hilbert space $L^{2}\left((0, T), \boldsymbol{H}_{0}(\mathbf{c u r l})\right) \cap H^{1}\left((0, T), \boldsymbol{L}^{2}(\Omega)\right)$ to the Hilbert space $L^{2}\left((0, T), \boldsymbol{H}_{0}(\mathbf{c u r l}) \cap \boldsymbol{H}(\operatorname{div}=0)\right) \cap H^{1}((0, T), \boldsymbol{H}(\operatorname{div}=0))$.

In the upcoming theorem, we will make use of the following set:

$\mathbb{M}:=\left\{(\boldsymbol{j}, \boldsymbol{e}) \in L^{2}\left((0, T), \boldsymbol{L}^{2}(\Omega)\right) \times L^{2}\left((0, T), \boldsymbol{L}^{2}(\Omega)\right)|| \boldsymbol{j}(x, t) \mid \leq j_{c}(x)\right.$ a.e. in $\Omega \times(0, T)$, $\boldsymbol{j}(x, t) \cdot \boldsymbol{e}(x, t)=j_{c}(x)|\boldsymbol{e}(x, t)|$ a.e. in $\left.\Omega \times(0, T)\right\}$.

We note that this set $\mathbb{M} \subset L^{2}\left((0, T), \boldsymbol{L}^{2}(\Omega)\right) \times L^{2}\left((0, T), \boldsymbol{L}^{2}(\Omega)\right)$ is maximal monotone (see Remark 1 in [13]).

TheOrem 3.2. Let $\left\{\boldsymbol{u}_{n}\right\}_{n=1}^{\infty}$ be a sequence in $H^{2}((0, T), \boldsymbol{H}(\operatorname{div}=0))$. For every $n \in \mathbb{N}$, let $\left(\boldsymbol{E}_{n}, \boldsymbol{H}_{n}, \boldsymbol{J}_{n}\right) \in L^{\infty}((0, T), D(\mathcal{A})) \cap W^{1, \infty}((0, T), \boldsymbol{X}) \times L^{2}\left((0, T), \boldsymbol{L}^{2}(\Omega)\right)$ denote the strong solution of (1.2) associated with $\boldsymbol{u}_{n} \in H^{2}((0, T), \boldsymbol{H}(\operatorname{div}=0))$. If $\boldsymbol{u}_{n} \rightarrow \boldsymbol{u}$ weakly in $H^{2}((0, T), \boldsymbol{H}(\operatorname{div}=0))$ as $n \rightarrow \infty$, then

$$
\begin{aligned}
& \left(\boldsymbol{E}_{n}, \boldsymbol{H}_{n}\right) \rightarrow(\boldsymbol{E}, \boldsymbol{H}) \text { weakly in } L^{2}((0, T), D(\mathcal{A})) \cap H^{1}((0, T), \boldsymbol{X}) \text { as } n \rightarrow \infty, \\
& \boldsymbol{J}_{n} \rightarrow \boldsymbol{J} \quad \text { weakly in } L^{2}\left((0, T), \boldsymbol{L}^{2}(\Omega)\right) \text { as } n \rightarrow \infty \text {, }
\end{aligned}
$$

where the triple $(\boldsymbol{E}, \boldsymbol{H}, \boldsymbol{J}) \in L^{\infty}((0, T), D(\mathcal{A})) \cap W^{1, \infty}((0, T), \boldsymbol{X}) \times L^{2}\left((0, T), \boldsymbol{L}^{2}(\Omega)\right)$ is the strong solution of (1.2) associated with the weak limit $\boldsymbol{u} \in H^{2}((0, T), \boldsymbol{H}(\operatorname{div}=0))$.

Proof. According to Lemma 2.4, the sequence $\left\{\left(\boldsymbol{E}_{n}, \boldsymbol{H}_{n}\right)\right\}_{n=1}^{\infty}$ is bounded in the Hilbert space $L^{2}((0, T), D(\mathcal{A})) \cap H^{1}((0, T), \boldsymbol{X})$. Moreover, by definition, the inequality $\left|\boldsymbol{J}_{n}(x, t)\right| \leq j_{c}(x)$ holds for a.e. $(x, t) \in \Omega \times(0, T)$ and all $n \in \mathbb{N}$. Thus, there exists a subsequence of $\left\{\left(\boldsymbol{E}_{n}, \boldsymbol{H}_{n}, \boldsymbol{J}_{n}\right)\right\}_{n=1}^{\infty}$, denoted by $\left\{\left(\boldsymbol{E}_{n_{k}}, \boldsymbol{H}_{n_{k}}, \boldsymbol{J}_{n_{k}}\right)\right\}_{k=1}^{\infty}$, such that

$$
\begin{array}{rll}
\left(\boldsymbol{E}_{n_{k}}, \boldsymbol{H}_{n_{k}}\right) & \rightarrow(\boldsymbol{E}, \boldsymbol{H}) & \text { weakly in } L^{2}((0, T), D(\mathcal{A})) \cap H^{1}((0, T), \boldsymbol{X}) \text { as } k \rightarrow \infty, \\
\boldsymbol{J}_{n_{k}} & \rightarrow \boldsymbol{J} & \text { weakly in } L^{2}\left((0, T), \boldsymbol{L}^{2}(\Omega)\right) \text { as } k \rightarrow \infty,
\end{array}
$$

for some $(\boldsymbol{E}, \boldsymbol{H}) \in L^{2}((0, T), D(\mathcal{A})) \cap H^{1}((0, T), \boldsymbol{X})$ and some $\boldsymbol{J} \in L^{2}\left((0, T), \boldsymbol{L}^{2}(\Omega)\right)$. 
According to Definition 2.2, for every $k \in \mathbb{N},\left(\boldsymbol{E}_{n_{k}}, \boldsymbol{H}_{n_{k}}\right)$ satisfies

$$
\left(\boldsymbol{E}_{n_{k}}, \boldsymbol{H}_{n_{k}}\right)(t)=\mathbb{T}_{t}\left(\boldsymbol{E}_{0}, \boldsymbol{H}_{0}\right)+\int_{0}^{t} \mathbb{T}_{t-s}\left(\epsilon^{-1}\left(\boldsymbol{u}_{n_{k}}(s)-\boldsymbol{J}_{n_{k}}(s)\right), 0\right) d s \forall t \in[0, T] .
$$

Hence, as $\boldsymbol{u}_{n_{k}} \rightarrow \boldsymbol{u}$ and $\boldsymbol{J}_{n_{k}} \rightarrow \boldsymbol{J}$ weakly in $L^{2}\left((0, T), \boldsymbol{L}^{2}(\Omega)\right)$, the weak limit $(\boldsymbol{E}, \boldsymbol{H})$ satisfies

$$
(\boldsymbol{E}, \boldsymbol{H})(t)=\mathbb{T}_{t}\left(\boldsymbol{E}_{0}, \boldsymbol{H}_{0}\right)+\int_{0}^{t} \mathbb{T}_{t-s}\left(\epsilon^{-1}(\boldsymbol{u}(s)-\boldsymbol{J}(s)), 0\right) d s \quad \forall t \in[0, T],
$$

and the following pointwise weak convergence is obtained:

$$
\left(\boldsymbol{E}_{n_{k}}, \boldsymbol{H}_{n_{k}}\right)(t) \rightarrow(\boldsymbol{E}, \boldsymbol{H})(t) \quad \text { weakly in } \boldsymbol{X} \text { as } k \rightarrow \infty \quad \forall t \in[0, T] .
$$

Let us show that $(\boldsymbol{E}, \boldsymbol{H}, \boldsymbol{J}) \in L^{\infty}((0, T), D(\mathcal{A})) \cap W^{1, \infty}((0, T), \boldsymbol{X}) \times L^{2}\left((0, T), \boldsymbol{L}^{2}(\Omega)\right)$ is the strong solution of (1.2) associated with the weak limit $\boldsymbol{u} \in H^{2}((0, T), \boldsymbol{H}(\operatorname{div}=0))$. In view of (3.6) and Lemma 2.4, we only have to verify that $(\boldsymbol{J}, \boldsymbol{E}) \in \mathbb{M}$. Making use of the energy balance equality (Lemma 2.6) in (3.5) and (3.6), we infer that

$$
\begin{aligned}
2 \int_{0}^{T}\left(\boldsymbol{J}_{n_{k}}(t), \boldsymbol{E}_{n_{k}}(t)\right)_{\boldsymbol{L}^{2}(\Omega)} d t= & -\left\|\left(\boldsymbol{E}_{n_{k}}, \boldsymbol{H}_{n_{k}}\right)(T)\right\|_{\boldsymbol{X}}^{2}+\left\|\left(\boldsymbol{E}_{0}, \boldsymbol{H}_{0}\right)\right\|_{\boldsymbol{X}}^{2} \\
& +2 \int_{0}^{T}\left(\boldsymbol{u}_{n_{k}}(t), \boldsymbol{E}_{n_{k}}(t)\right)_{\boldsymbol{L}^{2}(\Omega)} d t \\
= & -\left\|\left(\boldsymbol{E}_{n_{k}}, \boldsymbol{H}_{n_{k}}\right)(T)\right\|_{\boldsymbol{X}}^{2}+\|(\boldsymbol{E}, \boldsymbol{H})(T)\|_{\boldsymbol{X}}^{2}+2 \int_{0}^{T}(\boldsymbol{J}(t), \boldsymbol{E}(t))_{\boldsymbol{L}^{2}(\Omega)} d t \\
& +2 \int_{0}^{T}\left(\boldsymbol{u}_{n_{k}}(t), \boldsymbol{E}_{n_{k}}(t)\right)_{\boldsymbol{L}^{2}(\Omega)}-(\boldsymbol{u}(t), \boldsymbol{E}(t))_{\boldsymbol{L}^{2}(\Omega)} d t .
\end{aligned}
$$

Now, in view of Corollary 3.1, the weak convergence (3.4) implies that

$\boldsymbol{\pi} \boldsymbol{E}_{n_{k}} \rightarrow \boldsymbol{\pi} \boldsymbol{E} \quad$ weakly in $L^{2}\left((0, T), \boldsymbol{H}_{0}(\right.$ curl $\left.) \cap \boldsymbol{H}(\operatorname{div}=0)\right) \cap H^{1}((0, T), \boldsymbol{H}(\operatorname{div}=0))$,

as $k \rightarrow \infty$. On the other hand, as the injection $\boldsymbol{H}_{0}($ curl $) \cap \boldsymbol{H}(\operatorname{div}) \hookrightarrow \boldsymbol{L}^{2}(\Omega)$ is compact, the Aubin-Lions lemma yields the compactness of the injection

$$
L^{2}\left((0, T), \boldsymbol{H}_{0}(\mathbf{c u r l}) \cap \boldsymbol{H}(\operatorname{div})\right) \cap H^{1}\left((0, T), \boldsymbol{L}^{2}(\Omega)\right) \hookrightarrow L^{2}\left((0, T), \boldsymbol{L}^{2}(\Omega)\right),
$$

and so $\boldsymbol{\pi} \boldsymbol{E}_{n_{k}} \rightarrow \boldsymbol{\pi} \boldsymbol{E}$ strongly in $L^{2}\left((0, T), \boldsymbol{L}^{2}(\Omega)\right)$ as $k \rightarrow \infty$. From this strong convergence, it follows that

$$
\begin{aligned}
& \int_{0}^{T}\left(\boldsymbol{u}_{n_{k}}(t), \boldsymbol{E}_{n_{k}}(t)\right)_{\boldsymbol{L}^{2}(\Omega)} d t=\int_{0}^{T}\left(\boldsymbol{u}_{n_{k}}(t), \boldsymbol{\pi} \boldsymbol{E}_{n_{k}}(t)\right)_{\boldsymbol{L}^{2}(\Omega)} d t \\
& \rightarrow \int_{0}^{T}(\boldsymbol{u}(t), \boldsymbol{\pi} \boldsymbol{E}(t))_{\boldsymbol{L}^{2}(\Omega)} d t=\int_{0}^{T}(\boldsymbol{u}(t), \boldsymbol{E}(t))_{\boldsymbol{L}^{2}(\Omega)} d t \quad \text { as } k \rightarrow \infty,
\end{aligned}
$$

where we have also used (3.2) and the weak convergence $\boldsymbol{u}_{n_{k}} \rightarrow \boldsymbol{u}$ in $L^{2}\left((0, T), \boldsymbol{L}^{2}(\Omega)\right)$. The above convergence applied to (3.8) yields that 


$$
\begin{aligned}
& 2 \liminf _{k \rightarrow \infty} \int_{0}^{T}\left(\boldsymbol{J}_{n_{k}}(t), \boldsymbol{E}_{n_{k}}(t)\right)_{\boldsymbol{L}^{2}(\Omega)} d t \leq 2 \limsup _{k \rightarrow \infty} \int_{0}^{T}\left(\boldsymbol{J}_{n_{k}}(t), \boldsymbol{E}_{n_{k}}(t)\right)_{\boldsymbol{L}^{2}(\Omega)} d t \\
& \quad \leq \limsup _{k \rightarrow \infty}\left(-\left\|\left(\boldsymbol{E}_{n_{k}}, \boldsymbol{H}_{n_{k}}\right)(T)\right\|_{\boldsymbol{X}}^{2}\right)+\|(\boldsymbol{E}, \boldsymbol{H})(T)\|_{\boldsymbol{X}}^{2}+2 \int_{0}^{T}(\boldsymbol{J}(t), \boldsymbol{E}(t))_{\boldsymbol{L}^{2}(\Omega)} d t \\
& \quad=-\liminf _{k \rightarrow \infty}\left\|\left(\boldsymbol{E}_{n_{k}}, \boldsymbol{H}_{n_{k}}\right)(T)\right\|_{\boldsymbol{X}}^{2}+\|(\boldsymbol{E}, \boldsymbol{H})(T)\|_{\boldsymbol{X}}^{2}+2 \int_{0}^{T}(\boldsymbol{J}(t), \boldsymbol{E}(t))_{\boldsymbol{L}^{2}(\Omega)} d t
\end{aligned}
$$

and hence, by (3.7), it follows that

$$
\liminf _{k \rightarrow \infty} \int_{0}^{T}\left(\boldsymbol{J}_{n_{k}}(t), \boldsymbol{E}_{n_{k}}(t)\right)_{\boldsymbol{L}^{2}(\Omega)} d t \leq \int_{0}^{T}(\boldsymbol{J}(t), \boldsymbol{E}(t))_{\boldsymbol{L}^{2}(\Omega)} d t .
$$

Thus, since $\mathbb{M}$ is a maximal monotone set (Remark 1 in [13]) and $\left(\boldsymbol{J}_{n_{k}}, \boldsymbol{E}_{n_{k}}\right) \in \mathbb{M}$ holds for all $k \in \mathbb{N}$, the above inequality implies that $(\boldsymbol{J}, \boldsymbol{E}) \in \mathbb{M}$ (see Showalter $[20$, Proposition 1.6, p. 159]). In conclusion, $(\boldsymbol{E}, \boldsymbol{H}, \boldsymbol{J})$ is the strong solution of (1.2) associated with $\boldsymbol{u}$, and classical arguments imply that (3.4) is satisfied for the whole sequence $\left\{\left(\boldsymbol{E}_{n}, \boldsymbol{H}_{n}, \boldsymbol{J}_{n}\right)\right\}_{n=1}^{\infty}$.

An immediate consequence of Theorem 3.2 is the existence of an optimal solution for $(\mathrm{P})$, which we shall summarize in the upcoming corollary.

Definition 3.3. A triple $(\overline{\boldsymbol{E}}, \overline{\boldsymbol{H}}, \overline{\boldsymbol{u}}) \in \mathcal{F}_{\text {eas }}$ is called an optimal solution of $(\mathrm{P})$, if and only if $\mathcal{J}(\overline{\boldsymbol{E}}, \overline{\boldsymbol{H}}, \overline{\boldsymbol{u}}) \leq \mathcal{J}(\boldsymbol{E}, \boldsymbol{H}, \boldsymbol{u})$ holds for all $(\boldsymbol{E}, \boldsymbol{H}, \boldsymbol{u}) \in \mathcal{F}_{\text {eas }}$, where the feasible set $\mathcal{F}_{\text {eas }}$ associated with $(\mathrm{P})$ is given by

$$
\begin{aligned}
& \mathcal{F}_{\text {eas }}:=\left\{(\boldsymbol{E}, \boldsymbol{H}, \boldsymbol{u}) \in L^{\infty}((0, T), D(\mathcal{A})) \cap W^{1, \infty}((0, T), \boldsymbol{X}) \times H^{2}((0, T), \boldsymbol{H}(\operatorname{div}=0)) \mid\right. \\
& \exists \boldsymbol{J} \in L^{2}\left((0, T), \boldsymbol{L}^{2}(\Omega)\right) \text { s.t. }(\boldsymbol{E}, \boldsymbol{H}, \boldsymbol{J}) \text { is the strong solution of }(1.2) \text { associated with } \boldsymbol{u} .
\end{aligned}
$$

Corollary 3.4. The PDE-constrained optimization problem (P) admits an optimal solution $(\overline{\boldsymbol{E}}, \overline{\boldsymbol{H}}, \overline{\boldsymbol{u}}) \in \mathcal{F}_{\text {eas }}$.

4. Analysis of the penalized problem. In the following, let $(\overline{\boldsymbol{E}}, \overline{\boldsymbol{H}}, \overline{\boldsymbol{u}}) \in \mathcal{F}_{\text {eas }}$ be an arbitrarily fixed optimal solution of (P). For every $\gamma>0$, we consider

$$
\min \mathcal{J}\left(\boldsymbol{E}^{\gamma}, \boldsymbol{H}^{\gamma}, \boldsymbol{u}\right)+\frac{1}{2}\|\boldsymbol{u}-\overline{\boldsymbol{u}}\|_{H^{2}\left((0, T), \boldsymbol{L}^{2}(\Omega)\right)}^{2},
$$

subject to the semilinear hyperbolic evolution Maxwell equations

$$
\begin{cases}\epsilon \boldsymbol{E}_{t}^{\gamma}-\operatorname{curl} \boldsymbol{H}^{\gamma}+\varphi^{\gamma}\left(\cdot, \boldsymbol{E}^{\gamma}\right)=\boldsymbol{u} & \text { in } \Omega \times(0, T), \\ \mu \boldsymbol{H}_{t}^{\gamma}+\operatorname{curl} \boldsymbol{E}^{\gamma}=0 & \text { in } \Omega \times(0, T), \\ \boldsymbol{E}^{\gamma} \times \boldsymbol{n}=0 & \text { in } \partial \Omega \times(0, T), \\ \boldsymbol{E}^{\gamma}(\cdot, 0)=\boldsymbol{E}_{0} & \text { in } \Omega, \\ \boldsymbol{H}^{\gamma}(\cdot, 0)=\boldsymbol{H}_{0} & \text { in } \Omega\end{cases}
$$

and to the divergence-free constraint on the control

$$
\operatorname{div} \boldsymbol{u}=0 \quad \text { in } \Omega \times(0, T) .
$$

Here, the function $\varphi^{\gamma}: \Omega \times \mathbb{R}^{3} \rightarrow \mathbb{R}^{3}$ is defined as follows:

$$
\varphi^{\gamma}(x, y):=j_{c}(x) \frac{y}{\sqrt{\gamma^{-2}+|y|^{2}}} .
$$

Copyright $\odot$ by SIAM. Unauthorized reproduction of this article is prohibited. 
We denote by $\Phi^{\gamma}: \boldsymbol{L}^{2}(\Omega) \rightarrow \boldsymbol{L}^{2}(\Omega)$ the Nemytskii operator generated by $\varphi^{\gamma}$, i.e., $\Phi^{\gamma}(\boldsymbol{y}(\cdot))=\varphi^{\gamma}(\cdot, \boldsymbol{y}(\cdot))$ for every $\boldsymbol{y} \in \boldsymbol{L}^{2}(\Omega)$. For every fixed $\gamma>0$, the operator $\Phi^{\gamma}: \boldsymbol{L}^{2}(\Omega) \rightarrow \boldsymbol{L}^{2}(\Omega)$ is Lipschitz-continuous. Furthermore, it satisfies

$$
\left|\Phi^{\gamma}(\boldsymbol{y})(x)\right| \leq j_{c}(x) \quad \text { a.e. in } \Omega \quad \forall \boldsymbol{y} \in \boldsymbol{L}^{2}(\Omega), \forall \gamma>0 .
$$

Here, we recall that $j_{c}=\chi_{\Omega_{s c}} \mathfrak{j}_{c}$, where $\mathfrak{j}_{c} \in \mathbb{R}^{+}$denotes the critical current density of the superconductor $\Omega_{s c}$ (see section 1). Now, employing $\mathcal{A}$ and $\Phi^{\gamma}$, (4.1) can be equivalently formulated as the following Cauchy problem:

$$
\left\{\begin{aligned}
\left(\frac{d}{d t}-\mathcal{A}\right)\left(\boldsymbol{E}^{\gamma}, \boldsymbol{H}^{\gamma}\right)(t) & =\left(\epsilon^{-1}\left(\boldsymbol{u}(t)-\Phi^{\gamma}\left(\boldsymbol{E}^{\gamma}(t)\right)\right), 0\right), \quad t \in(0, T), \\
\left(\boldsymbol{E}^{\gamma}, \boldsymbol{H}^{\gamma}\right)(0) & =\left(\boldsymbol{E}_{0}, \boldsymbol{H}_{0}\right) .
\end{aligned}\right.
$$

Definition 4.1. Let $\gamma>0$ and $\boldsymbol{u} \in L^{1}\left((0, T), \boldsymbol{L}^{2}(\Omega)\right)$. A pair $\left(\boldsymbol{E}^{\gamma}, \boldsymbol{H}^{\gamma}\right) \in$ $\mathcal{C}([0, T], \boldsymbol{X})$ is called a mild solution of (4.1) associated with $\boldsymbol{u}$ if and only if

$$
\left(\boldsymbol{E}^{\gamma}, \boldsymbol{H}^{\gamma}\right)(t)=\mathbb{T}_{t}\left(\boldsymbol{E}_{0}, \boldsymbol{H}_{0}\right)+\int_{0}^{t} \mathbb{T}_{t-s}\left(\epsilon^{-1}\left(\boldsymbol{u}(s)-\Phi^{\gamma}\left(\boldsymbol{E}^{\gamma}(s)\right)\right), 0\right) d s
$$

holds for all $t \in[0, T]$.

Remark 4.2. According to the classical result by Ball [3], $\left(\boldsymbol{E}^{\gamma}, \boldsymbol{H}^{\gamma}\right) \in \mathcal{C}([0, T], \boldsymbol{X})$ is a mild solution of (4.1) associated with $\boldsymbol{u}$ if and only if it satisfies the following the weak formulation for (4.1):

$$
\left\{\begin{array}{l}
\frac{d}{d t} \int_{\Omega} \epsilon \boldsymbol{E}^{\gamma}(t) \cdot \boldsymbol{v} d x-\int_{\Omega} \boldsymbol{H}^{\gamma}(t) \cdot \operatorname{curl} \boldsymbol{v} d x+\int_{\Omega} \Phi^{\gamma}\left(\boldsymbol{E}^{\gamma}(t)\right) \cdot \boldsymbol{v} d x=\int_{\Omega} \boldsymbol{u}(t) \cdot \boldsymbol{v} d x, \\
\frac{d}{d t} \int_{\Omega} \mu \boldsymbol{H}^{\gamma}(t) \cdot \boldsymbol{w} d x+\int_{\Omega} \boldsymbol{E}^{\gamma}(t) \cdot \operatorname{curl} \boldsymbol{w} d x=0 \\
\text { for a.e. } t \in(0, T) \text { and all }(\boldsymbol{v}, \boldsymbol{w}) \in \boldsymbol{H}_{0}(\operatorname{curl}) \times \boldsymbol{H}(\operatorname{curl}), \\
\left(\boldsymbol{E}^{\gamma}, \boldsymbol{H}^{\gamma}\right)(0)=\left(\boldsymbol{E}_{0}, \boldsymbol{H}_{0}\right)
\end{array}\right.
$$

and, for every $(\boldsymbol{v}, \boldsymbol{w}) \in \boldsymbol{H}_{0}($ curl $) \times \boldsymbol{H}($ curl $)$, the mapping

$$
t \mapsto \int_{\Omega} \epsilon \boldsymbol{E}^{\gamma}(t) \cdot \boldsymbol{v}+\mu \boldsymbol{H}^{\gamma}(t) \cdot \boldsymbol{w} d x
$$

is absolutely continuous from $[0, T]$ to $\mathbb{R}$, and so it is a.e. differentiable in $(0, T)$.

Lemma 4.3. For every $\gamma>0$ and $\boldsymbol{u} \in L^{1}\left((0, T), \boldsymbol{L}^{2}(\Omega)\right)$, (4.1) admits a unique mild solution $\left(\boldsymbol{E}^{\gamma}, \boldsymbol{H}^{\gamma}\right) \in \mathcal{C}([0, T], \boldsymbol{X})$.

Proof. Let $\gamma>0$ and $\boldsymbol{u} \in L^{1}\left((0, T), \boldsymbol{L}^{2}(\Omega)\right)$. Introducing the function

$$
g \in \mathcal{C}([0, T], \boldsymbol{X}), \quad g(t):=\mathbb{T}_{t}\left(\boldsymbol{E}_{0}, \boldsymbol{H}_{0}\right)+\int_{0}^{t} \mathbb{T}_{t-s}\left(\epsilon^{-1} \boldsymbol{u}(s), 0\right) d s \quad \forall t \in[0, T],
$$

we see that (4.5) is equivalent to

$$
\left(\boldsymbol{E}^{\gamma}, \boldsymbol{H}^{\gamma}\right)(t)=g(t)+\int_{0}^{t} \mathbb{T}_{t-s} \alpha\left(\left(\boldsymbol{E}^{\gamma}, \boldsymbol{H}^{\gamma}\right)(s)\right) d s \quad \forall t \in[0, T]
$$

where $\alpha: \boldsymbol{X} \rightarrow \boldsymbol{X}, \alpha(\boldsymbol{v}, \boldsymbol{w}):=-\left(\epsilon^{-1} \Phi^{\gamma}(\boldsymbol{v}), 0\right)$. Since $\alpha: \boldsymbol{X} \rightarrow \boldsymbol{X}$ is Lipschitzcontinuous and $g \in \mathcal{C}([0, T], \boldsymbol{X})$, a classical contraction argument (cf. [16, Corollary 1.3 , p. 185]) implies that the integral equation (4.6) admits a unique solution $\left(\boldsymbol{E}^{\gamma}, \boldsymbol{H}^{\gamma}\right) \in \mathcal{C}([0, T], \boldsymbol{X})$. 
Lemma 4.4. Let $\boldsymbol{u} \in L^{1}\left((0, T), \boldsymbol{L}^{2}(\Omega)\right)$. Then, for every $\gamma>0$, the mild solution $\left(\boldsymbol{E}^{\gamma}, \boldsymbol{H}^{\gamma}\right) \in \mathcal{C}([0, T], \boldsymbol{X})$ of (4.1) associated with $\boldsymbol{u}$ satisfies

$$
\left\|\left(\boldsymbol{E}^{\gamma}, \boldsymbol{H}^{\gamma}\right)\right\|_{\mathcal{C}([0, T], \boldsymbol{X})} \leq\left\|\left(\boldsymbol{E}_{0}, \boldsymbol{H}_{0}\right)\right\|_{\boldsymbol{X}}+2 \underline{\epsilon}^{-\frac{1}{2}}\|\boldsymbol{u}\|_{L^{1}\left((0, T), \boldsymbol{L}^{2}(\Omega)\right)} .
$$

Proof. In view of (4.5), the energy balance equality (Lemma 2.6) implies

$$
\begin{aligned}
\left\|\left(\boldsymbol{E}^{\gamma}, \boldsymbol{H}^{\gamma}\right)(t)\right\|_{\boldsymbol{X}}^{2}= & \left\|\left(\boldsymbol{E}_{0}, \boldsymbol{H}_{0}\right)\right\|_{\boldsymbol{X}}^{2}+2 \int_{0}^{t}\left[\left(\boldsymbol{u}(s), \boldsymbol{E}^{\gamma}(s)\right)_{\boldsymbol{L}^{2}(\Omega)}\right. \\
& \left.-\left(\Phi^{\gamma}\left(\boldsymbol{E}^{\gamma}(s)\right), \boldsymbol{E}^{\gamma}(s)\right)_{\boldsymbol{L}^{2}(\Omega)}\right] d s \quad \forall t \in[0, T] .
\end{aligned}
$$

Thus, since $\left(\Phi^{\gamma}(\boldsymbol{v}), \boldsymbol{v}\right)_{\boldsymbol{L}^{2}(\Omega)} \geq 0$ holds for all $\boldsymbol{v} \in \boldsymbol{L}^{2}(\Omega)$, it follows that

$$
\begin{aligned}
& \left\|\left(\boldsymbol{E}^{\gamma}, \boldsymbol{H}^{\gamma}\right)(t)\right\|_{\boldsymbol{X}}^{2} \leq\left\|\left(\boldsymbol{E}_{0}, \boldsymbol{H}_{0}\right)\right\|_{\boldsymbol{X}}^{2}+2 \int_{0}^{t}\left(\boldsymbol{u}(s), \boldsymbol{E}^{\gamma}(s)\right)_{\boldsymbol{L}^{2}(\Omega)} d s \\
& \quad \leq\left\|\left(\boldsymbol{E}_{0}, \boldsymbol{H}_{0}\right)\right\|_{\boldsymbol{X}}^{2}+2 \underline{\epsilon}^{-\frac{1}{2}} \max _{t \in[0, T]}\left\|\boldsymbol{E}^{\gamma}(t)\right\|_{\boldsymbol{L}_{\epsilon}^{2}(\Omega)}\|\boldsymbol{u}\|_{L^{1}\left((0, T), \boldsymbol{L}^{2}(\Omega)\right)} \\
& \quad \leq\left\|\left(\boldsymbol{E}^{\gamma}, \boldsymbol{H}^{\gamma}\right)\right\|_{\mathcal{C}([0, T], \boldsymbol{X})}\left(\left\|\left(\boldsymbol{E}_{0}, \boldsymbol{H}_{0}\right)\right\|_{\boldsymbol{X}}+2 \underline{\epsilon}^{-\frac{1}{2}}\|\boldsymbol{u}\|_{L^{1}\left((0, T), \boldsymbol{L}^{2}(\Omega)\right)}\right) \forall t \in[0, T] .
\end{aligned}
$$

In conclusion, $\left\|\left(\boldsymbol{E}^{\gamma}, \boldsymbol{H}^{\gamma}\right)\right\|_{\mathcal{C}([0, T], \boldsymbol{X})} \leq\left\|\left(\boldsymbol{E}_{0}, \boldsymbol{H}_{0}\right)\right\|_{\boldsymbol{X}}+2 \underline{\epsilon}^{-\frac{1}{2}}\|\boldsymbol{u}\|_{L^{1}\left((0, T), \boldsymbol{L}^{2}(\Omega)\right)}$.

Remark 4.5. The previous results consider only $\boldsymbol{u} \in L^{1}\left((0, T), \boldsymbol{L}^{2}(\Omega)\right)$ for the applied current source. In the upcoming lemma, we demonstrate that if $\boldsymbol{u} \in W^{1, \infty}((0, T)$, $\boldsymbol{L}^{2}(\Omega)$ ), then the mild solution of (4.1) turns out to be the (classical) solution of (4.4) or equivalently (4.1). At this point we should notice that every function $\boldsymbol{u} \in$ $W^{1, \infty}\left((0, T), \boldsymbol{L}^{2}(\Omega)\right)$ is, possibly after a modification on a set of $[0, T]$ with measure zero, Lipschitz-continuous from $[0, T]$ to $\boldsymbol{L}^{2}(\Omega)$ such that it makes sense to consider the Cauchy problem (4.4) pointwise for all $t \in[0, T]$.

Lemma 4.6. Let $\gamma>0$ and $\boldsymbol{u} \in W^{1, \infty}\left((0, T), \boldsymbol{L}^{2}(\Omega)\right)$. Then, the mild solution $\left(\boldsymbol{E}^{\gamma}, \boldsymbol{H}^{\gamma}\right)$ of (4.1) enjoys the regularity property $\left(\boldsymbol{E}^{\gamma}, \boldsymbol{H}^{\gamma}\right) \in \mathcal{C}([0, T], D(\mathcal{A})) \cap$ $\mathcal{C}^{1}([0, T], \boldsymbol{X})$ and satisfies (4.4) for all $t \in[0, T]$, i.e., it is the solution of (4.1).

Proof. As $\Phi^{\gamma}: \boldsymbol{L}^{2}(\Omega) \rightarrow \boldsymbol{L}^{2}(\Omega)$ is Lipschitz-continuous, $\boldsymbol{u} \in W^{1, \infty}\left((0, T), \boldsymbol{L}^{2}(\Omega)\right)$, and $\left(\boldsymbol{E}_{0}, \boldsymbol{H}_{0}\right) \in D(\mathcal{A})$, classical arguments (cf. the proof of [16, Theorem 1.6, p. 189]) imply that the mild solution of (4.4) is Lipschitz-continuous, i.e., $\left(\boldsymbol{E}^{\gamma}, \boldsymbol{H}^{\gamma}\right) \in$ $\mathcal{C}^{0,1}([0, T], \boldsymbol{X})$. Thus, since the operator $\Phi^{\gamma}: \boldsymbol{L}^{2}(\Omega) \rightarrow \boldsymbol{L}^{2}(\Omega)$ is Lipschitz-continuous and $\boldsymbol{X}$ is reflexive, it follows that the right-hand side of (4.4) satisfies $\left(\epsilon^{-1}(\boldsymbol{u}-\right.$ $\left.\left.\Phi^{\gamma}\left(\boldsymbol{E}^{\gamma}\right)\right), 0\right) \in W^{1, \infty}((0, T), \boldsymbol{X})$. By this regularity property and $\left(\boldsymbol{E}_{0}, \boldsymbol{H}_{0}\right) \in D(\mathcal{A})$, we may apply $[10$, Corollary 7.6, p. 440$]$ to deduce that $\left(\boldsymbol{E}^{\gamma}, \boldsymbol{H}^{\gamma}\right) \in \mathcal{C}([0, T], D(\mathcal{A})) \cap$ $\mathcal{C}^{1}([0, T], \boldsymbol{X})$ is the (classical) solution of (4.4).

Lemma 4.7. Let $\gamma>0$ and $\boldsymbol{u} \in W^{1, \infty}\left((0, T), \boldsymbol{L}^{2}(\Omega)\right)$. Then, the solution $\left(\boldsymbol{E}^{\gamma}, \boldsymbol{H}^{\gamma}\right)$ $\in \mathcal{C}([0, T], D(\mathcal{A})) \cap \mathcal{C}^{1}([0, T], \boldsymbol{X})$ of (4.1) associated with $\boldsymbol{u}$ satisfies

$$
\left\|\left(\boldsymbol{E}^{\gamma}, \boldsymbol{H}^{\gamma}\right)\right\|_{\mathcal{C}([0, T], D(\mathcal{A})) \cap \mathcal{C}^{1}([0, T], \boldsymbol{X})} \leq c\left(\|\boldsymbol{u}\|_{W^{1, \infty}\left((0, T), \boldsymbol{L}^{2}(\Omega)\right)}+1\right),
$$

with a constant $c>0$, independent of $\gamma, \boldsymbol{u}$, and $\left(\boldsymbol{E}^{\gamma}, \boldsymbol{H}^{\gamma}\right)$.

Proof. Let $t \in(0, T)$ and $h>0$ such that $t+h \in(0, T)$. According to (4.5), we have that

Copyright $@$ by SIAM. Unauthorized reproduction of this article is prohibited. 


$$
\begin{aligned}
\left(\boldsymbol{E}^{\gamma}, \boldsymbol{H}^{\gamma}\right)(t+h)= & \mathbb{T}_{t+h}\left(\boldsymbol{E}_{0}, \boldsymbol{H}_{0}\right)+\int_{0}^{t+h} \mathbb{T}_{t+h-s}\left(\epsilon^{-1}\left(\boldsymbol{u}(s)-\Phi^{\gamma}\left(\boldsymbol{E}^{\gamma}(s)\right)\right), 0\right) d s, \\
= & \mathbb{T}_{t+h}\left(\boldsymbol{E}_{0}, \boldsymbol{H}_{0}\right)+\int_{0}^{h} \mathbb{T}_{t+h-s}\left(\epsilon^{-1}\left(\boldsymbol{u}(s)-\Phi^{\gamma}\left(\boldsymbol{E}^{\gamma}(s)\right)\right), 0\right) d s \\
& +\int_{h}^{t+h} \mathbb{T}_{t+h-s}\left(\epsilon^{-1}\left(\boldsymbol{u}(s)-\Phi^{\gamma}\left(\boldsymbol{E}^{\gamma}(s)\right)\right), 0\right) d s, \\
= & \mathbb{T}_{t}\left(\mathbb{T}_{h}\left(\boldsymbol{E}_{0}, \boldsymbol{H}_{0}\right)+\int_{0}^{h} \mathbb{T}_{h-s}\left(\epsilon^{-1}\left(\boldsymbol{u}(s)-\Phi^{\gamma}\left(\boldsymbol{E}^{\gamma}(s)\right)\right), 0\right) d s\right) \\
& +\int_{0}^{t} \mathbb{T}_{t-s}\left(\epsilon^{-1}\left(\boldsymbol{u}(s+h)-\Phi^{\gamma}\left(\boldsymbol{E}^{\gamma}(s+h)\right)\right), 0\right) d s,
\end{aligned}
$$

and hence, taking again (4.5) into account, it follows that

$$
\begin{aligned}
& \frac{\left(\boldsymbol{E}^{\gamma}, \boldsymbol{H}^{\gamma}\right)(t+h)-\left(\boldsymbol{E}^{\gamma}, \boldsymbol{H}^{\gamma}\right)(t)}{h} \\
&= \mathbb{T}_{t}\left(\frac{\mathbb{T}_{h}\left(\boldsymbol{E}_{0}, \boldsymbol{H}_{0}\right)-\left(\boldsymbol{E}_{0}, \boldsymbol{H}_{0}\right)}{h}+\frac{1}{h} \int_{0}^{h} \mathbb{T}_{h-s}\left(\epsilon^{-1}\left(\boldsymbol{u}(s)-\Phi^{\gamma}\left(\boldsymbol{E}^{\gamma}(s)\right)\right), 0\right) d s\right) \\
& \quad+\frac{1}{h} \int_{0}^{t} \mathbb{T}_{t-s}\left(\epsilon^{-1}\left((\boldsymbol{u}(s+h)-\boldsymbol{u}(s))+\Phi^{\gamma}\left(\boldsymbol{E}^{\gamma}(s)\right)-\Phi^{\gamma}\left(\boldsymbol{E}^{\gamma}(s+h)\right)\right), 0\right) d s .
\end{aligned}
$$

Then, the energy balance equality (Lemma 2.6) implies

$$
\begin{aligned}
& \| \frac{\left(\boldsymbol{E}^{\gamma}, \boldsymbol{H}^{\gamma}\right)(t+h)-\left(\boldsymbol{E}^{\gamma}, \boldsymbol{H}^{\gamma}\right)(t)}{h} \|_{\boldsymbol{X}}^{2} \\
&=\left\|\frac{\mathbb{T}_{h}\left(\boldsymbol{E}_{0}, \boldsymbol{H}_{0}\right)-\left(\boldsymbol{E}_{0}, \boldsymbol{H}_{0}\right)}{h}+\frac{1}{h} \int_{0}^{h} \mathbb{T}_{h-s}\left(\epsilon^{-1}\left(\boldsymbol{u}(s)-\Phi^{\gamma}\left(\boldsymbol{E}^{\gamma}(s)\right)\right), 0\right) d s\right\|_{\boldsymbol{X}}^{2} \\
& \quad+2 \int_{0}^{t}\left[\left(\frac{\boldsymbol{u}(s+h)-\boldsymbol{u}(s)}{h}, \frac{\boldsymbol{E}^{\gamma}(s+h)-\boldsymbol{E}^{\gamma}(s)}{h}\right)_{\boldsymbol{L}^{2}(\Omega)}\right. \\
&\left.\quad+\frac{1}{h^{2}}\left(\Phi^{\gamma}\left(\boldsymbol{E}^{\gamma}(s)\right)-\Phi^{\gamma}\left(\boldsymbol{E}^{\gamma}(s+h)\right), \boldsymbol{E}^{\gamma}(s+h)-\boldsymbol{E}^{\gamma}(s)\right)_{\boldsymbol{L}^{2}(\Omega)}\right] d s \\
&=: I(h)+I I(h) .
\end{aligned}
$$

We estimate the first term $I(h)$ as follows:

$$
\begin{aligned}
& I(h) \leq\left(\left\|\frac{\mathbb{T}_{h}\left(\boldsymbol{E}_{0}, \boldsymbol{H}_{0}\right)-\left(\boldsymbol{E}_{0}, \boldsymbol{H}_{0}\right)}{h}\right\|_{\boldsymbol{X}}+\frac{1}{h} \int_{0}^{h}\left\|\left(\epsilon^{-1}\left(\boldsymbol{u}(s)-\Phi^{\gamma}\left(\boldsymbol{E}^{\gamma}(s)\right)\right), 0\right)\right\|_{\boldsymbol{X}} d s\right)^{2} \\
& \underbrace{\leq}_{(4.3)}\left(\left\|\frac{\mathbb{T}_{h}\left(\boldsymbol{E}_{0}, \boldsymbol{H}_{0}\right)-\left(\boldsymbol{E}_{0}, \boldsymbol{H}_{0}\right)}{h}\right\|_{\boldsymbol{X}}+\underline{\epsilon}^{-\frac{1}{2}}\|\boldsymbol{u}\|_{L^{\infty}\left((0, T), \boldsymbol{L}^{2}(\Omega)\right)}+\underline{\epsilon}^{-\frac{1}{2}} \mathfrak{j}_{c}\left|\Omega_{s c}\right|^{\frac{1}{2}}\right)^{2} .
\end{aligned}
$$

As $\left(\boldsymbol{E}_{0}, \boldsymbol{H}_{0}\right) \in D(\mathcal{A})$, we can pass to the limit $h \rightarrow 0$ in the above inequality and obtain

$$
\limsup _{h \rightarrow 0} I(h) \leq\left(\left\|\mathcal{A}\left(\boldsymbol{E}_{0}, \boldsymbol{H}_{0}\right)\right\|_{\boldsymbol{X}}+\underline{\epsilon}^{-\frac{1}{2}}\|\boldsymbol{u}\|_{L^{\infty}\left((0, T), \boldsymbol{L}^{2}(\Omega)\right)}+\underline{\epsilon}^{-\frac{1}{2}} \mathbf{j}_{c}\left|\Omega_{s c}\right|^{\frac{1}{2}}\right)^{2} .
$$

Copyright (? by SIAM. Unauthorized reproduction of this article is prohibited. 
On the other hand, as $\left(\Phi^{\gamma}(\boldsymbol{v})-\Phi^{\gamma}(\boldsymbol{w}), \boldsymbol{v}-\boldsymbol{w}\right)_{\boldsymbol{L}^{2}(\Omega)} \geq 0$ holds for all $\boldsymbol{v}, \boldsymbol{w} \in \boldsymbol{L}^{2}(\Omega)$, the second term $I I(h)$ can be estimated as follows:

$$
\begin{aligned}
I I(h)= & 2 \int_{0}^{t}\left[\left(\frac{\boldsymbol{u}(s+h)-\boldsymbol{u}(s)}{h}, \frac{\boldsymbol{E}^{\gamma}(s+h)-\boldsymbol{E}^{\gamma}(s)}{h}\right)_{\boldsymbol{L}^{2}(\Omega)}\right. \\
& \left.-\frac{1}{h^{2}}\left(\Phi^{\gamma}\left(\boldsymbol{E}^{\gamma}(s+h)\right)-\Phi^{\gamma}\left(\boldsymbol{E}^{\gamma}(s)\right), \boldsymbol{E}^{\gamma}(s+h)-\boldsymbol{E}^{\gamma}(s)\right)_{\boldsymbol{L}^{2}(\Omega)}\right] d s \\
\leq & 2 \int_{0}^{t}\left(\frac{\boldsymbol{u}(s+h)-\boldsymbol{u}(s)}{h}, \frac{\boldsymbol{E}^{\gamma}(s+h)-\boldsymbol{E}^{\gamma}(s)}{h}\right)_{\boldsymbol{L}^{2}(\Omega)} d s \\
\leq & 2 T \underline{\epsilon}^{-\frac{1}{2}}\left\|\frac{d}{d t} \boldsymbol{u}\right\|_{L^{\infty}\left((0, T), \boldsymbol{L}^{2}(\Omega)\right)}\left\|\frac{d}{d t} \boldsymbol{E}^{\gamma}\right\|_{\mathcal{C}\left([0, T], \boldsymbol{L}_{\epsilon}^{2}(\Omega)\right)}+\frac{1}{2}\left\|\frac{d}{d t} \boldsymbol{E}^{\gamma}\right\|_{\mathcal{C}\left([0, T], \boldsymbol{L}_{\epsilon}^{2}(\Omega)\right)}^{2} . \\
\leq & 2 T^{2} \underline{\epsilon}^{-1}\left\|\frac{d}{d t} \boldsymbol{u}\right\|_{L^{\infty}\left((0, T), \boldsymbol{L}^{2}(\Omega)\right)}^{2}
\end{aligned}
$$

Concluding from (4.8)-(4.10) and since $\left(\boldsymbol{E}^{\gamma}, \boldsymbol{H}^{\gamma}\right) \in \mathcal{C}^{1}([0, T], \boldsymbol{X})$, we obtain

$$
\begin{aligned}
\left\|\frac{d}{d t}\left(\boldsymbol{E}^{\gamma}, \boldsymbol{H}^{\gamma}\right)(t)\right\|_{\boldsymbol{X}}^{2}=\lim _{h \downarrow 0}\left\|\frac{\left(\boldsymbol{E}^{\gamma}, \boldsymbol{H}^{\gamma}\right)(t+h)-\left(\boldsymbol{E}^{\gamma}, \boldsymbol{H}^{\gamma}\right)(t)}{h}\right\|_{\boldsymbol{X}}^{2} \\
\leq\left(\left\|\mathcal{A}\left(\boldsymbol{E}_{0}, \boldsymbol{H}_{0}\right)\right\|_{\boldsymbol{X}}+\underline{\epsilon}^{-\frac{1}{2}}\|\boldsymbol{u}\|_{L^{\infty}\left((0, T), \boldsymbol{L}^{2}(\Omega)\right)}+\underline{\epsilon}^{-\frac{1}{2}} \mathfrak{j}_{c}\left|\Omega_{s c}\right|^{\frac{1}{2}}\right)^{2} \\
\quad+2 T^{2} \underline{\epsilon}^{-1}\left\|\frac{d}{d t} \boldsymbol{u}\right\|_{L^{\infty}\left((0, T), \boldsymbol{L}^{2}(\Omega)\right)}^{2}+\frac{1}{2}\left\|\frac{d}{d t} \boldsymbol{E}^{\gamma}\right\|_{\mathcal{C}\left([0, T], \boldsymbol{L}_{\epsilon}^{2}(\Omega)\right)}^{2} \quad \forall t \in(0, T),
\end{aligned}
$$

from which it follows that

$$
\begin{aligned}
\left\|\frac{d}{d t}\left(\boldsymbol{E}^{\gamma}, \boldsymbol{H}^{\gamma}\right)\right\|_{\mathcal{C}([0, T], \boldsymbol{X})}^{2} \leq & 2\left(\left\|\mathcal{A}\left(\boldsymbol{E}_{0}, \boldsymbol{H}_{0}\right)\right\|_{\boldsymbol{X}}+\underline{\epsilon}^{-\frac{1}{2}}\|\boldsymbol{u}\|_{L^{\infty}\left((0, T), \boldsymbol{L}^{2}(\Omega)\right)}\right. \\
& \left.+\underline{\epsilon}^{-\frac{1}{2}} \mathfrak{j}_{c}\left|\Omega_{s c}\right|^{\frac{1}{2}}\right)^{2}+4 T^{2} \underline{\epsilon}^{-1}\left\|\frac{d}{d t} \boldsymbol{u}\right\|_{L^{\infty}\left((0, T), \boldsymbol{L}^{2}(\Omega)\right)}^{2}
\end{aligned}
$$

Finally, as $\left(\boldsymbol{E}^{\gamma}, \boldsymbol{H}^{\gamma}\right) \in \mathcal{C}([0, T], D(\mathcal{A})) \cap \mathcal{C}^{1}([0, T], \boldsymbol{X})$ satisfies (4.4) for all $t \in[0, T]$, we obtain that

$$
\left\|\mathcal{A}\left(\boldsymbol{E}^{\gamma}, \boldsymbol{H}^{\gamma}\right)\right\|_{\mathcal{C}([0, T], \boldsymbol{X})} \leq\left\|\frac{d}{d t}\left(\boldsymbol{E}^{\gamma}, \boldsymbol{H}^{\gamma}\right)\right\|_{\mathcal{C}([0, T], \boldsymbol{X})}+\underline{\epsilon}^{-\frac{1}{2}}\left(\|\boldsymbol{u}\|_{\mathcal{C}\left([0, T], \boldsymbol{L}^{2}(\Omega)\right)}+\mathfrak{j}_{c}\left|\Omega_{s c}\right|^{\frac{1}{2}}\right) .
$$

In conclusion, the assertion follows from the above inequality along with (4.11) and Lemma 4.4.

Let us close this section by proving an existence result for $\left(\mathrm{P}_{\gamma}^{\bar{u}}\right)$.

THEOREM 4.8. For every $\gamma>0$, the penalized problem $\left(\mathrm{P}_{\gamma}^{\bar{u}}\right)$ admits an optimal solution $\left(\overline{\boldsymbol{E}}^{\gamma}, \overline{\boldsymbol{H}}^{\gamma}, \overline{\boldsymbol{u}}^{\gamma}\right) \in \mathcal{C}([0, T], D(\mathcal{A})) \cap \mathcal{C}^{1}([0, T], \boldsymbol{X}) \times H^{2}((0, T), \boldsymbol{H}(\operatorname{div}=0))$.

Proof. Let $\gamma>0$. By classical arguments, it suffices to prove the following statement: If $\boldsymbol{u}_{n} \rightarrow \boldsymbol{u}$ weakly in $H^{2}((0, T), \boldsymbol{H}(\operatorname{div}=0))$ as $n \rightarrow \infty$, then

$$
\left(\boldsymbol{E}_{n}^{\gamma}, \boldsymbol{H}_{n}^{\gamma}\right) \rightarrow\left(\boldsymbol{E}^{\gamma}, \boldsymbol{H}^{\gamma}\right) \text { weakly in } L^{2}((0, T), \boldsymbol{X}) \text { as } n \rightarrow \infty,
$$

where, for every $n \in \mathbb{N},\left(\boldsymbol{E}_{n}^{\gamma}, \boldsymbol{H}_{n}^{\gamma}\right) \in \mathcal{C}([0, T], D(\mathcal{A})) \cap \mathcal{C}^{1}([0, T], \boldsymbol{X})$ denotes the solution of (4.1) associated with $\boldsymbol{u}_{n}$, and $\left(\boldsymbol{E}^{\gamma}, \boldsymbol{H}^{\gamma}\right) \in \mathcal{C}([0, T], D(\mathcal{A})) \cap \mathcal{C}^{1}([0, T], \boldsymbol{X})$ denotes the 
solution of (4.1) associated with $\boldsymbol{u}$. The proof is completely analogous to the one for Theorem 3.2. For the convenience of the reader, we provide the main steps of the proof. By virtue of Lemma 4.7 and (4.3), there exists a subsequence of $\left\{\left(\boldsymbol{E}_{n}^{\gamma}, \boldsymbol{H}_{n}^{\gamma}\right)\right\}_{n=1}^{\infty}$, denoted by $\left\{\left(\boldsymbol{E}_{n_{j}}^{\gamma}, \boldsymbol{H}_{n_{j}}^{\gamma}\right)\right\}_{j=1}^{\infty}$, such that

$$
\begin{aligned}
& \left(\boldsymbol{E}_{n_{j}}^{\gamma}, \boldsymbol{H}_{n_{j}}^{\gamma}\right) \rightarrow(\boldsymbol{E}, \boldsymbol{H}) \quad \text { weakly in } L^{2}((0, T), D(\mathcal{A})) \cap H^{1}((0, T), \boldsymbol{X}) \text { as } j \rightarrow \infty, \\
& \Phi^{\gamma}\left(\boldsymbol{E}_{n_{j}}^{\gamma}\right) \rightarrow \boldsymbol{J} \quad \text { weakly in } L^{2}\left((0, T), \boldsymbol{L}^{2}(\Omega)\right) \text { as } j \rightarrow \infty,
\end{aligned}
$$

for some $(\boldsymbol{E}, \boldsymbol{H}) \in L^{2}((0, T), D(\mathcal{A})) \cap H^{1}((0, T), \boldsymbol{X})$ and some $\boldsymbol{J} \in L^{2}\left((0, T), \boldsymbol{L}^{2}(\Omega)\right)$. Then, we use analogous arguments as in the proof of Theorem 3.2 based on the energy balance equality (Lemma 2.6) and the Helmholtz projection to deduce that

$$
\liminf _{j \rightarrow \infty} \int_{0}^{T}\left(\boldsymbol{E}_{n_{j}}^{\gamma}(t), \Phi^{\gamma}\left(\boldsymbol{E}_{n_{j}}^{\gamma}(t)\right)\right)_{\boldsymbol{L}^{2}(\Omega)} d t \leq \int_{0}^{T}(\boldsymbol{E}(t), \boldsymbol{J}(t))_{\boldsymbol{L}^{2}(\Omega)} d t .
$$

Since $\Phi^{\gamma}: L^{2}\left((0, T), \boldsymbol{L}^{2}(\Omega)\right) \rightarrow L^{2}\left((0, T), \boldsymbol{L}^{2}(\Omega)\right)$ is monotone and continuous, it is maximal monotone, and so (4.13) implies $\boldsymbol{J}=\Phi^{\gamma}(\boldsymbol{E})$. Consequently, $(\boldsymbol{E}, \boldsymbol{H})$ is the solution to (4.1) associated with $\boldsymbol{u}$, i.e., $(\boldsymbol{E}, \boldsymbol{H})=\left(\boldsymbol{E}^{\gamma}, \boldsymbol{H}^{\gamma}\right)$. Finally, as $\left(\boldsymbol{E}^{\gamma}, \boldsymbol{H}^{\gamma}\right)$ is independent of the subsequence $\left\{\left(\boldsymbol{E}_{n_{j}}^{\gamma}, \boldsymbol{H}_{n_{j}}^{\gamma}\right)\right\}_{j=1}^{\infty}$, classical arguments imply (4.12).

4.1. Convergence analysis. In this section, we prove the weak convergence of the solution of $\left(\mathrm{P}_{\gamma}^{\overline{\boldsymbol{u}}}\right)$ toward $(\overline{\boldsymbol{E}}, \overline{\boldsymbol{H}}, \overline{\boldsymbol{u}})$ as $\gamma \rightarrow \infty$.

Lemma 4.9. Let $\left\{\boldsymbol{u}^{\gamma}\right\}_{\gamma>0} \subset H^{2}((0, T), \boldsymbol{H}(\operatorname{div}=0))$. For every $\gamma>0$, let $\left(\boldsymbol{E}^{\gamma}, \boldsymbol{H}^{\gamma}\right)$ $\in \mathcal{C}([0, T], D(\mathcal{A})) \cap \mathcal{C}^{1}([0, T], \boldsymbol{X})$ denote the solution of (4.1) associated with $\boldsymbol{u}^{\gamma}$ and we set

$$
\boldsymbol{J}^{\gamma}:=\Phi^{\gamma}\left(\boldsymbol{E}^{\gamma}\right)=j_{c} \frac{\boldsymbol{E}^{\gamma}}{\sqrt{\gamma^{-2}+\left|\boldsymbol{E}^{\gamma}\right|^{2}}}
$$

If $\boldsymbol{u}^{\gamma} \rightarrow \boldsymbol{u}$ weakly in $H^{2}((0, T), \boldsymbol{H}(\operatorname{div}=0))$ as $\gamma \rightarrow \infty$, then

$$
\begin{array}{rlrl}
\left(\boldsymbol{E}^{\gamma}, \boldsymbol{H}^{\gamma}\right) & \rightarrow(\boldsymbol{E}, \boldsymbol{H}) & & \text { weakly in } L^{2}((0, T), D(\mathcal{A})) \cap H^{1}((0, T), \boldsymbol{X}) \text { as } \gamma \rightarrow \infty, \\
\left(\boldsymbol{E}^{\gamma}, \boldsymbol{H}^{\gamma}\right)(t) & \rightarrow(\boldsymbol{E}, \boldsymbol{H})(t) & & \text { weakly in } \boldsymbol{X} \text { as } \gamma \rightarrow \infty \text { for all } t \in[0, T], \\
\boldsymbol{J}^{\gamma} & \rightarrow \boldsymbol{J} & \text { weakly in } L^{2}\left((0, T), \boldsymbol{L}^{2}(\Omega)\right) \text { as } \gamma \rightarrow \infty,
\end{array}
$$

where the triple $(\boldsymbol{E}, \boldsymbol{H}, \boldsymbol{J}) \in L^{\infty}((0, T), D(\mathcal{A})) \cap W^{1, \infty}((0, T), \boldsymbol{X}) \times L^{2}\left((0, T), \boldsymbol{L}^{2}(\Omega)\right)$ is the strong solution of (1.2) associated with the weak limit $\boldsymbol{u} \in H^{2}((0, T), \boldsymbol{H}(\operatorname{div}=0))$.

Proof. In view of Lemma 4.7 and since $\left|\boldsymbol{J}^{\gamma}(x, t)\right| \leq j_{c}(x)$ holds for almost all $(x, t) \in \Omega \times(0, T)$ and all $\gamma>0$, there exists a subsequence of $\left\{\left(\boldsymbol{E}^{\gamma}, \boldsymbol{H}^{\gamma}, \boldsymbol{J}^{\gamma}\right)\right\}_{\gamma>0}$, denoted by $\left\{\left(\boldsymbol{E}^{\gamma_{n}}, \boldsymbol{H}^{\gamma_{n}}, \boldsymbol{J}^{\gamma_{n}}\right)\right\}_{n=1}^{\infty}$ (with $\gamma_{n} \rightarrow \infty$ as $\left.n \rightarrow \infty\right)$, such that

$$
\begin{aligned}
\left(\boldsymbol{E}^{\gamma_{n}}, \boldsymbol{H}^{\gamma_{n}}\right) & \rightarrow(\widetilde{\boldsymbol{E}}, \widetilde{\boldsymbol{H}}) & & \text { weakly in } L^{2}((0, T), D(\mathcal{A})) \cap H^{1}((0, T), \boldsymbol{X}), \\
\boldsymbol{J}^{\gamma_{n}} & \rightarrow \widetilde{\boldsymbol{J}} & & \text { weakly in } L^{2}\left((0, T), \boldsymbol{L}^{2}(\Omega)\right),
\end{aligned}
$$

as $n \rightarrow \infty$, for some $(\widetilde{\boldsymbol{E}}, \widetilde{\boldsymbol{H}}) \in L^{2}((0, T), D(\mathcal{A})) \cap H^{1}((0, T), \boldsymbol{X})$ and some $\widetilde{\boldsymbol{J}} \in$ $L^{2}\left((0, T), \boldsymbol{L}^{2}(\Omega)\right)$ satisfying

$$
|\widetilde{\boldsymbol{J}}(x, t)| \leq j_{c}(x) \quad \text { a.e. in } \Omega \times(0, T) .
$$

Copyright $@$ by SIAM. Unauthorized reproduction of this article is prohibited. 
By the weak convergence (4.15)-(4.16) and since $\boldsymbol{u}^{\gamma} \rightarrow \boldsymbol{u}$ weakly in $L^{2}\left((0, T), \boldsymbol{L}^{2}(\Omega)\right)$ as $\gamma \rightarrow \infty$, it follows from (4.5) that

$$
(\widetilde{\boldsymbol{E}}, \widetilde{\boldsymbol{H}})(t)=\mathbb{T}_{t}\left(\boldsymbol{E}_{0}, \boldsymbol{H}_{0}\right)+\int_{0}^{t} \mathbb{T}_{t-s}\left(\epsilon^{-1}(\boldsymbol{u}(s)-\widetilde{\boldsymbol{J}}(s)), 0\right) d s \quad \forall t \in[0, T]
$$

and

$$
\left(\boldsymbol{E}^{\gamma_{n}}, \boldsymbol{H}^{\gamma_{n}}\right)(t) \rightarrow(\widetilde{\boldsymbol{E}}, \widetilde{\boldsymbol{H}})(t) \quad \text { weakly in } \boldsymbol{X} \text { as } n \rightarrow \infty \quad \forall t \in[0, T] .
$$

Let us show that $(\widetilde{\boldsymbol{E}}, \widetilde{\boldsymbol{H}}, \widetilde{\boldsymbol{J}}) \in L^{\infty}((0, T), D(\mathcal{A})) \cap W^{1, \infty}((0, T), \boldsymbol{X}) \times L^{2}\left((0, T), \boldsymbol{L}^{2}(\Omega)\right)$ is the strong solution of (1.2) associated with the weak limit $\boldsymbol{u} \in H^{2}((0, T), \boldsymbol{H}(\operatorname{div}=0))$. In view of Lemma 2.4 along with (4.17) and (4.18), we only need to prove

$$
\widetilde{\boldsymbol{J}}(x, t) \cdot \widetilde{\boldsymbol{E}}(x, t)=j_{c}(x)|\widetilde{\boldsymbol{E}}(x, t)| \quad \text { a.e. in } \Omega \times(0, T) .
$$

To this aim, we define $\mathbf{R}^{\gamma_{n}}:=\boldsymbol{E}^{\gamma_{n}} \frac{\left|\boldsymbol{E}^{\gamma_{n}}\right|}{\sqrt{\gamma_{n}^{-2}+\left|\boldsymbol{E}^{\gamma_{n}}\right|^{2}}}$. Due to our construction, it holds for almost all $(x, t) \in \Omega \times(0, T)$ that

$$
\boldsymbol{J}^{\gamma_{n}}(x, t) \cdot \boldsymbol{E}^{\gamma_{n}}(x, t) \underbrace{=}_{(4.14)} j_{c}(x) \frac{\left|\boldsymbol{E}^{\gamma_{n}}(x, t)\right|^{2}}{\sqrt{\gamma_{n}^{-2}+\left|\boldsymbol{E}^{\gamma_{n}}(x, t)\right|^{2}}}=j_{c}(x)\left|\mathbf{R}^{\gamma_{n}}(x, t)\right| .
$$

Moreover, the inequality $\left\|\boldsymbol{E}^{\gamma_{n}}-\mathbf{R}^{\gamma_{n}}\right\|_{L^{2}\left((0, T), L^{2}(\Omega)\right)} \leq \gamma_{n}^{-1}(T|\Omega|)^{1 / 2}$ holds for all $n \in \mathbb{N}$, and hence (4.15) implies

$$
\mathbf{R}^{\gamma_{n}} \rightarrow \widetilde{\boldsymbol{E}} \quad \text { weakly in } L^{2}\left((0, T), \boldsymbol{L}^{2}(\Omega)\right) \text { as } n \rightarrow \infty .
$$

Let now $\tau \in \mathbb{R}^{+}$. The weak convergence (4.22) implies

$$
\begin{aligned}
& \int_{0}^{T} \int_{\Omega} j_{c}(x) \frac{|\widetilde{\boldsymbol{E}}(x, t)|^{2}}{|\widetilde{\boldsymbol{E}}(x, t)|+\tau} d x d t=\lim _{n \rightarrow \infty} \int_{0}^{T} \int_{\Omega} j_{c}(x) \frac{\widetilde{\boldsymbol{E}}(x, t)}{|\widetilde{\boldsymbol{E}}(x, t)|+\tau} \cdot \mathbf{R}^{\gamma_{n}}(x, t) d x d t \\
&=\liminf _{n \rightarrow \infty} \int_{0}^{T} \int_{\Omega} j_{c}(x) \frac{\widetilde{\boldsymbol{E}}(x, t)}{|\widetilde{\boldsymbol{E}}(x, t)|+\tau} \cdot \mathbf{R}^{\gamma_{n}}(x, t) d x d t \\
& \leq \liminf _{n \rightarrow \infty} \int_{0}^{T} \int_{\Omega} j_{c}(x)\left|\mathbf{R}^{\gamma_{n}}(x, t)\right| d x d t \\
& \underbrace{=}_{(4.21)} \liminf _{n \rightarrow \infty} \int_{0}^{T} \int_{\Omega} \boldsymbol{J}^{\gamma_{n}}(x, t) \cdot \boldsymbol{E}^{\gamma_{n}}(x, t) d x d t .
\end{aligned}
$$

Passing to the limit $\tau \rightarrow 0$, it follows that

$$
\int_{0}^{T} \int_{\Omega} j_{c}(x)|\widetilde{\boldsymbol{E}}(x, t)| d x d t \leq \liminf _{n \rightarrow \infty} \int_{0}^{T} \int_{\Omega} \boldsymbol{J}^{\gamma_{n}}(x, t) \cdot \boldsymbol{E}^{\gamma_{n}}(x, t) d x d t
$$

To estimate the right-hand side of the above inequality, we make use of the energy balance equality (Lemma 2.6) in (4.5) and (4.18):

Copyright (? by SIAM. Unauthorized reproduction of this article is prohibited. 


$$
\begin{aligned}
2 \int_{0}^{T}\left(\boldsymbol{J}^{\gamma_{n}}(t), \boldsymbol{E}^{\gamma_{n}}(t)\right)_{\boldsymbol{L}^{2}(\Omega)} d t= & -\left\|\left(\boldsymbol{E}^{\gamma_{n}}, \boldsymbol{H}^{\gamma_{n}}\right)(T)\right\|_{\boldsymbol{X}}^{2}+\left\|\left(\boldsymbol{E}_{0}, \boldsymbol{H}_{0}\right)\right\|_{\boldsymbol{X}}^{2} \\
& +2 \int_{0}^{T}\left(\boldsymbol{u}^{\gamma_{n}}(t), \boldsymbol{E}^{\gamma_{n}}(t)\right)_{\boldsymbol{L}^{2}(\Omega)} d t \\
= & -\left\|\left(\boldsymbol{E}^{\gamma_{n}}, \boldsymbol{H}^{\gamma_{n}}\right)(T)\right\|_{\boldsymbol{X}}^{2}+\|(\widetilde{\boldsymbol{E}}, \widetilde{\boldsymbol{H}})(T)\|_{\boldsymbol{X}}^{2}+2 \int_{0}^{T}(\widetilde{\boldsymbol{J}}(t), \widetilde{\boldsymbol{E}}(t)) d t \\
+ & 2 \int_{0}^{T}\left(\boldsymbol{u}^{\gamma_{n}}(t)-\boldsymbol{u}(t), \boldsymbol{E}^{\gamma_{n}}(t)\right)_{\boldsymbol{L}^{2}(\Omega)} d t .
\end{aligned}
$$

Furthermore, analogously as in the proof of Theorem 3.2, we have that

$$
\int_{0}^{T}\left(\boldsymbol{u}^{\gamma_{n}}(t)-\boldsymbol{u}(t), \boldsymbol{E}^{\gamma_{n}}(t)\right)_{\boldsymbol{L}^{2}(\Omega)} d t \underbrace{=}_{(3.2)} \int_{0}^{T}\left(\boldsymbol{u}^{\gamma_{n}}(t)-\boldsymbol{u}(t), \boldsymbol{\pi} \boldsymbol{E}^{\gamma_{n}}(t)\right)_{\boldsymbol{L}^{2}(\Omega)} d t \rightarrow 0,
$$

as $n \rightarrow \infty$. Then, applying the above convergence and (4.19) to (4.24), it follows that

$$
\liminf _{n \rightarrow \infty} \int_{0}^{T} \int_{\Omega} \boldsymbol{J}^{\gamma_{n}}(x, t) \cdot \boldsymbol{E}^{\gamma_{n}}(x, t) d x d t \leq \int_{0}^{T} \int_{\Omega} \widetilde{\boldsymbol{J}}(x, t) \cdot \widetilde{\boldsymbol{E}}(x, t) d x d t .
$$

As a result of this inequality in combination with (4.23), we obtain

$$
\int_{0}^{T} \int_{\Omega}\left(j_{c}(x)|\widetilde{\boldsymbol{E}}(x, t)|-\widetilde{\boldsymbol{J}}(x, t) \cdot \widetilde{\boldsymbol{E}}(x, t)\right) d x d t \leq 0 .
$$

Furthermore,

$$
j_{c}(x)|\widetilde{\boldsymbol{E}}(x, t)|-\widetilde{\boldsymbol{J}}(x, t) \cdot \widetilde{\boldsymbol{E}}(x, t) \underbrace{\geq}_{(4.17)} 0 \text { a.e. in } \Omega \times(0, T) .
$$

Combining the above two inequalities yields finally (4.20). In conclusion, $(\widetilde{\boldsymbol{E}}, \widetilde{\boldsymbol{H}}, \widetilde{\boldsymbol{J}})=$ $(\boldsymbol{E}, \boldsymbol{H}, \boldsymbol{J})$ is the strong solution of (1.2) associated with $\boldsymbol{u}$. In particular, the weak limit is independent of the subsequence $\left\{\left(\boldsymbol{E}^{\gamma_{n}}, \boldsymbol{H}^{\gamma_{n}}, \boldsymbol{J}^{\gamma_{n}}\right)\right\}_{n=1}^{\infty}$. Thus, classical arguments imply that the weak convergence (4.15)-(4.16) is satisfied for the whole sequence $\left\{\left(\boldsymbol{E}^{\gamma}, \boldsymbol{H}^{\gamma}, \boldsymbol{J}^{\gamma}\right)\right\}_{\gamma>0}$. This completes the proof.

LEMmA 4.10. Let $\boldsymbol{u} \in H^{2}((0, T), \boldsymbol{H}(\operatorname{div}=0))$ and $(\boldsymbol{E}, \boldsymbol{H}, \boldsymbol{J}) \in L^{\infty}((0, T), D(\mathcal{A})) \cap$ $W^{1, \infty}((0, T), \boldsymbol{X}) \times L^{2}\left((0, T), \boldsymbol{L}^{2}(\Omega)\right)$ denote the strong solution of (1.2) associated with $\boldsymbol{u}$. Furthermore, for each $\gamma>0$, let $\left(\boldsymbol{E}^{\gamma}, \boldsymbol{H}^{\gamma}\right) \in \mathcal{C}([0, T], D(\mathcal{A})) \cap \mathcal{C}^{1}([0, T], \boldsymbol{X})$ denote the solution of (4.1) associated with $\boldsymbol{u}$. Then,

$$
\left(\boldsymbol{E}^{\gamma}, \boldsymbol{H}^{\gamma}\right) \rightarrow(\boldsymbol{E}, \boldsymbol{H}) \quad \text { strongly in } L^{2}((0, T), \boldsymbol{X}) \text { as } \gamma \rightarrow \infty .
$$

Proof. For every $\gamma>0$, we set $\boldsymbol{J}^{\gamma}:=\Phi^{\gamma}\left(\boldsymbol{E}^{\gamma}\right)=j_{c} \frac{\boldsymbol{E}^{\gamma}}{\sqrt{\gamma^{-2}+\left|\boldsymbol{E}^{\gamma}\right|^{2}}}$. Then, Definitions 2.2 and 4.1 imply that

$$
\left(\boldsymbol{E}^{\gamma}-\boldsymbol{E}, \boldsymbol{H}^{\gamma}-\boldsymbol{H}\right)(t)=\int_{0}^{t} \mathbb{T}_{t-s}\left(\epsilon^{-1}\left(\boldsymbol{J}(s)-\boldsymbol{J}^{\gamma}(s)\right), 0\right) d s \quad \forall t \in[0, T],
$$

and hence, by the energy balance equality (Lemma 2.6), it follows that

Copyright $@$ ( ) by SIAM. Unauthorized reproduction of this article is prohibited. 


$$
\begin{aligned}
\left\|\left(\boldsymbol{E}^{\gamma}-\boldsymbol{E}, \boldsymbol{H}^{\gamma}-\boldsymbol{H}\right)(t)\right\|_{\boldsymbol{X}}^{2}= & 2 \int_{0}^{t}\left(\boldsymbol{J}(s)-\boldsymbol{J}^{\gamma}(s), \boldsymbol{E}^{\gamma}(s)-\boldsymbol{E}(s)\right)_{\boldsymbol{L}^{2}(\Omega)} d s \\
= & 2 \int_{0}^{t}\left(\boldsymbol{J}(s), \boldsymbol{E}^{\gamma}(s)-\boldsymbol{E}(s)\right)_{\boldsymbol{L}^{2}(\Omega)}+\left(\boldsymbol{J}^{\gamma}(s), \boldsymbol{E}(s)\right)_{\boldsymbol{L}^{2}(\Omega)} d s \\
& -2 \int_{0}^{t}\left(\boldsymbol{J}^{\gamma}(s), \boldsymbol{E}^{\gamma}(s)\right)_{\boldsymbol{L}^{2}(\Omega)} d s \quad \forall t \in[0, T] .
\end{aligned}
$$

Exploiting the weak convergence property from Lemma 4.9, we have

$$
\begin{aligned}
\lim _{\gamma \rightarrow \infty} & \left(2 \int_{0}^{t}\left(\boldsymbol{J}(s), \boldsymbol{E}^{\gamma}(s)-\boldsymbol{E}(s)\right)_{\boldsymbol{L}^{2}(\Omega)}+\left(\boldsymbol{J}^{\gamma}(s), \boldsymbol{E}(s)\right)_{\boldsymbol{L}^{2}(\Omega)} d s\right) \\
& =2 \int_{0}^{t}(\boldsymbol{J}(s), \boldsymbol{E}(s))_{\boldsymbol{L}^{2}(\Omega)} d s=2 \int_{0}^{t} \int_{\Omega} j_{c}(x)|\boldsymbol{E}(x, s)| d x d s \quad \forall t \in[0, T] .
\end{aligned}
$$

On the other hand, according to (4.23), we also have

$$
\liminf _{\gamma \rightarrow \infty} 2 \int_{0}^{t}\left(\boldsymbol{J}^{\gamma}(s), \boldsymbol{E}^{\gamma}(s)\right)_{\boldsymbol{L}^{2}(\Omega)} d s \geq 2 \int_{0}^{t} \int_{\Omega} j_{c}(x)|\boldsymbol{E}(x, s)| d x d s \quad \forall t \in[0, T] .
$$

Now, from (4.25)-(4.27), it follows that

$$
\begin{array}{r}
0 \leq \liminf _{\gamma \rightarrow \infty}\left\|\left(\boldsymbol{E}^{\gamma}-\boldsymbol{E}, \boldsymbol{H}^{\gamma}-\boldsymbol{H}\right)(t)\right\|_{\boldsymbol{X}}^{2} \leq \limsup _{\gamma \rightarrow \infty}\left\|\left(\boldsymbol{E}^{\gamma}-\boldsymbol{E}, \boldsymbol{H}^{\gamma}-\boldsymbol{H}\right)(t)\right\|_{\boldsymbol{X}}^{2} \\
\leq \limsup _{\gamma \rightarrow \infty}\left(2 \int_{0}^{t}\left(\boldsymbol{J}(s), \boldsymbol{E}^{\gamma}(s)-\boldsymbol{E}(s)\right)_{\boldsymbol{L}^{2}(\Omega)}+\left(\boldsymbol{J}^{\gamma}(s), \boldsymbol{E}(s)\right)_{\boldsymbol{L}^{2}(\Omega)} d s\right) \\
+\limsup _{\gamma \rightarrow \infty}\left(-2 \int_{0}^{t}\left(\boldsymbol{J}^{\gamma}(s), \boldsymbol{E}^{\gamma}(s)\right)_{\boldsymbol{L}^{2}(\Omega)} d s\right) \\
=2 \int_{0}^{t} \int_{\Omega} j_{c}(x)|\boldsymbol{E}(x, s)| d x d s-\liminf _{\gamma \rightarrow \infty}\left(2 \int_{0}^{t}\left(\boldsymbol{J}^{\gamma}(s), \boldsymbol{E}^{\gamma}(s)\right)_{\boldsymbol{L}^{2}(\Omega)} d s\right) \\
\leq 0 \quad \forall t \in[0, T] .
\end{array}
$$

Consequently, $\left(\boldsymbol{E}^{\gamma}, \boldsymbol{H}^{\gamma}\right)(t) \rightarrow(\boldsymbol{E}, \boldsymbol{H})(t)$ strongly in $\boldsymbol{X}$ for all $t \in[0, T]$. By this pointwise convergence together with (4.7), we may apply Lebesgue's dominated convergence theorem to deduce the strong convergence

$$
\left(\boldsymbol{E}^{\gamma}, \boldsymbol{H}^{\gamma}\right) \rightarrow(\boldsymbol{E}, \boldsymbol{H}) \quad \text { strongly in } L^{2}((0, T), \boldsymbol{X}) \text { as } \gamma \rightarrow \infty,
$$

which completes the proof.

Now, we have all the ingredients at hand to prove the weak convergence of the solution of $\left(\mathrm{P}_{\gamma}^{\bar{u}}\right)$ toward the optimal solution $(\overline{\boldsymbol{E}}, \overline{\boldsymbol{H}}, \overline{\boldsymbol{u}})$ of $(\mathrm{P})$ as $\gamma \rightarrow \infty$.

Theorem 4.11. Let $\left\{\left(\overline{\boldsymbol{E}}^{\gamma}, \overline{\boldsymbol{H}}^{\gamma}, \overline{\boldsymbol{u}}^{\gamma}\right)\right\}_{\gamma>0} \quad \subset \quad \mathcal{C}([0, T], D(\mathcal{A})) \cap \mathcal{C}^{1}([0, T], \boldsymbol{X}) \times$ $H^{2}((0, T), \boldsymbol{H}(\operatorname{div}=0))$ denote a sequence of optimal solutions of $\left(\mathrm{P}_{\gamma}^{\bar{u}}\right)$. Then,

$$
\begin{aligned}
& \left(\overline{\boldsymbol{E}}^{\gamma}, \overline{\boldsymbol{H}}^{\gamma}\right) \rightarrow(\overline{\boldsymbol{E}}, \overline{\boldsymbol{H}}) \quad \text { weakly in } L^{2}((0, T), D(\mathcal{A})) \cap H^{1}((0, T), \boldsymbol{X}) \text { as } \gamma \rightarrow \infty \text {, } \\
& \overline{\boldsymbol{u}}^{\gamma} \rightarrow \overline{\boldsymbol{u}} \quad \text { weakly in } H^{2}((0, T), \boldsymbol{H}(\operatorname{div}=0)) \text { as } \gamma \rightarrow \infty \text {. }
\end{aligned}
$$

Copyright (? by SIAM. Unauthorized reproduction of this article is prohibited. 
Proof. The assertion follows from Lemmas 4.9 and 4.10. For every $\gamma>0$, let $\left(\boldsymbol{E}_{\overline{\boldsymbol{u}}}^{\gamma}, \boldsymbol{H}_{\overline{\boldsymbol{u}}}^{\gamma}\right) \in \mathcal{C}([0, T], D(\mathcal{A})) \cap \mathcal{C}^{1}([0, T], \boldsymbol{X})$ denote the solution of (4.1) associated with $\overline{\boldsymbol{u}}$. Lemma 4.10 implies that

$$
\left(\boldsymbol{E}_{\overline{\boldsymbol{u}}}^{\gamma}, \boldsymbol{H}_{\overline{\boldsymbol{u}}}^{\gamma}\right) \rightarrow(\overline{\boldsymbol{E}}, \overline{\boldsymbol{H}}) \quad \text { strongly in } L^{2}((0, T), \boldsymbol{X}) \text { as } \gamma \rightarrow \infty .
$$

Furthermore, since $\left(\boldsymbol{E}_{\overline{\boldsymbol{u}}}^{\gamma}, \boldsymbol{H}_{\overline{\boldsymbol{u}}}^{\gamma}, \overline{\boldsymbol{u}}\right)$ is feasible for $\left(\mathrm{P}_{\gamma}^{\overline{\boldsymbol{u}}}\right)$ for every $\gamma>0$, we deduce that

$$
\mathcal{J}\left(\overline{\boldsymbol{E}}^{\gamma}, \overline{\boldsymbol{H}}^{\gamma}, \overline{\boldsymbol{u}}^{\gamma}\right)+\frac{1}{2}\left\|\overline{\boldsymbol{u}}^{\gamma}-\overline{\boldsymbol{u}}\right\|_{H^{2}\left((0, T), \boldsymbol{L}^{2}(\Omega)\right)}^{2} \leq \mathcal{J}\left(\boldsymbol{E}_{\overline{\boldsymbol{u}}}^{\gamma}, \boldsymbol{H}_{\overline{\boldsymbol{u}}}^{\gamma}, \overline{\boldsymbol{u}}\right) \quad \forall \gamma>0 .
$$

Thus, there exists a subsequence of $\left\{\overline{\boldsymbol{u}}^{\gamma}\right\}_{\gamma>0}$, which we denote by $\left\{\overline{\boldsymbol{u}}^{\gamma_{n}}\right\}_{n=1}^{\infty}$ (with $\gamma_{n} \rightarrow \infty$ as $\left.n \rightarrow \infty\right)$, such that

$$
\overline{\boldsymbol{u}}^{\gamma_{n}} \rightarrow \widetilde{\boldsymbol{u}} \quad \text { weakly in } H^{2}((0, T), \boldsymbol{H}(\operatorname{div}=0)) \text { as } n \rightarrow \infty,
$$

for some $\widetilde{\boldsymbol{u}} \in H^{2}((0, T), \boldsymbol{H}(\operatorname{div}=0))$. Now, Lemma 4.9 implies

$$
\left(\overline{\boldsymbol{E}}^{\gamma_{n}}, \overline{\boldsymbol{H}}^{\gamma_{n}}\right) \rightarrow(\widetilde{\boldsymbol{E}}, \widetilde{\boldsymbol{H}}) \quad \text { weakly in } L^{2}((0, T), D(\mathcal{A})) \cap H^{1}((0, T), \boldsymbol{X}) \text { as } n \rightarrow \infty
$$

with $(\widetilde{\boldsymbol{E}}, \widetilde{\boldsymbol{H}}, \widetilde{\boldsymbol{u}}) \in \mathcal{F}_{\text {eas }}$ (see Definition 3.3 for the feasible set $\mathcal{F}_{\text {eas }}$ ). The functional $F: L^{2}((0, T), \boldsymbol{X}) \times H^{2}\left((0, T), \boldsymbol{L}^{2}(\Omega)\right) \rightarrow \mathbb{R}$,

$$
F(\boldsymbol{E}, \boldsymbol{H}, \boldsymbol{u}):=\mathcal{J}(\boldsymbol{E}, \boldsymbol{H}, \boldsymbol{u})+\frac{1}{2}\|\boldsymbol{u}-\overline{\boldsymbol{u}}\|_{H^{2}\left((0, T), \boldsymbol{L}^{2}(\Omega)\right)}^{2},
$$

is convex and continuous, and hence it is sequentially weakly lower semicontinuous. Then, applying (4.28), (4.30), and (4.31) to (4.29), we obtain

$$
\mathcal{J}(\widetilde{\boldsymbol{E}}, \widetilde{\boldsymbol{H}}, \widetilde{\boldsymbol{u}})+\frac{1}{2}\|\widetilde{\boldsymbol{u}}-\overline{\boldsymbol{u}}\|_{H^{2}\left((0, T), \boldsymbol{L}^{2}(\Omega)\right)}^{2} \leq \mathcal{J}(\overline{\boldsymbol{E}}, \overline{\boldsymbol{H}}, \overline{\boldsymbol{u}}) .
$$

Thus, since $(\widetilde{\boldsymbol{E}}, \widetilde{\boldsymbol{H}}, \widetilde{\boldsymbol{u}}) \in \mathcal{F}_{\text {eas }}$ and $(\overline{\boldsymbol{E}}, \overline{\boldsymbol{H}}, \overline{\boldsymbol{u}})$ is an optimal solution of (P), it follows that

$$
\mathcal{J}(\widetilde{\boldsymbol{E}}, \widetilde{\boldsymbol{H}}, \widetilde{\boldsymbol{u}})+\frac{1}{2}\|\widetilde{\boldsymbol{u}}-\overline{\boldsymbol{u}}\|_{H^{2}\left((0, T), L^{2}(\Omega)\right)}^{2} \leq \mathcal{J}(\overline{\boldsymbol{E}}, \overline{\boldsymbol{H}}, \overline{\boldsymbol{u}}) \leq \mathcal{J}(\widetilde{\boldsymbol{E}}, \widetilde{\boldsymbol{H}}, \widetilde{\boldsymbol{u}}),
$$

and consequently $\widetilde{\boldsymbol{u}}=\overline{\boldsymbol{u}}$ and $(\widetilde{\boldsymbol{E}}, \widetilde{\boldsymbol{H}})=(\overline{\boldsymbol{E}}, \overline{\boldsymbol{H}})$. Since the weak limit is independent of the subsequence $\left\{\left(\overline{\boldsymbol{E}}^{\gamma_{n}}, \overline{\boldsymbol{H}}^{\gamma_{n}}, \overline{\boldsymbol{u}}^{\gamma_{n}}\right)\right\}_{n=1}^{\infty}$, classical arguments imply that the weak convergence (4.30)-(4.31) is satisfied for the whole sequence $\left\{\left(\overline{\boldsymbol{E}}^{\gamma}, \overline{\boldsymbol{H}}^{\gamma}, \overline{\boldsymbol{u}}^{\gamma}\right)\right\}_{\gamma>0}$.

4.2. Optimality system for $\left(\mathrm{P}_{\gamma}^{\bar{u}}\right)$. In the following, let $\gamma>0$ be arbitrarily fixed. We denote by

$$
G_{\gamma}: L^{1}\left((0, T), \boldsymbol{L}^{2}(\Omega)\right) \rightarrow \mathcal{C}([0, T], \boldsymbol{X}), \quad \boldsymbol{u} \mapsto(\boldsymbol{E}, \boldsymbol{H}),
$$

the mild solution operator associated with (4.1). In other words, for every $\boldsymbol{u} \in$ $L^{1}\left((0, T), \boldsymbol{L}^{2}(\Omega)\right), G_{\gamma}(\boldsymbol{u})=(\boldsymbol{E}, \boldsymbol{H}) \in \mathcal{C}([0, T], \boldsymbol{X})$ is given by the unique solution of the integral equation

$$
(\boldsymbol{E}, \boldsymbol{H})(t)=\mathbb{T}_{t}\left(\boldsymbol{E}_{0}, \boldsymbol{H}_{0}\right)+\int_{0}^{t} \mathbb{T}_{t-s}\left(\epsilon^{-1}\left(\boldsymbol{u}(s)-\varphi^{\gamma}(\cdot, \boldsymbol{E}(s))\right), 0\right) d s \quad \forall t \in[0, T] .
$$

See (4.2) for the definition of the function $\varphi^{\gamma}: \Omega \times \mathbb{R}^{3} \rightarrow \mathbb{R}^{3}$. 
Lemma 4.12. For all $\boldsymbol{u}_{1}, \boldsymbol{u}_{2} \in L^{1}\left((0, T), \boldsymbol{L}^{2}(\Omega)\right)$, it holds that

$$
\left\|G_{\gamma}\left(\boldsymbol{u}_{1}\right)-G_{\gamma}\left(\boldsymbol{u}_{2}\right)\right\|_{\mathcal{C}([0, T], \boldsymbol{X})} \leq 2 \underline{\epsilon}^{-1 / 2}\left\|\boldsymbol{u}_{1}-\boldsymbol{u}_{2}\right\|_{L^{1}\left((0, T), L^{2}(\Omega)\right)} .
$$

In other words, $G_{\gamma}: L^{1}\left((0, T), \boldsymbol{L}^{2}(\Omega)\right) \rightarrow \mathcal{C}([0, T], \boldsymbol{X})$ is Lipschitz-continuous with the Lipschitz constant $L=2 \underline{\epsilon}^{-1 / 2}$, independent of $\gamma$.

Proof. Let $\boldsymbol{u}_{1}, \boldsymbol{u}_{2} \in L^{1}\left((0, T), \boldsymbol{L}^{2}(\Omega)\right)$ and we set $\left(\boldsymbol{E}_{1}, \boldsymbol{H}_{1}\right)=G_{\gamma}\left(\boldsymbol{u}_{1}\right)$ and $\left(\boldsymbol{E}_{2}, \boldsymbol{H}_{2}\right)=G_{\gamma}\left(\boldsymbol{u}_{2}\right)$. By definition, we have

$$
\begin{aligned}
& \left(\boldsymbol{E}_{1}-\boldsymbol{E}_{2}, \boldsymbol{H}_{1}-\boldsymbol{H}_{2}\right)(t) \\
& \quad=\int_{0}^{t} \mathbb{T}_{t-s}\left(\epsilon^{-1}\left(\boldsymbol{u}_{1}(s)-\boldsymbol{u}_{2}(s)-\varphi^{\gamma}\left(\cdot, \boldsymbol{E}_{1}(s)\right)+\varphi^{\gamma}\left(\cdot, \boldsymbol{E}_{2}(s)\right)\right), 0\right) d s \quad \forall t \in[0, T] .
\end{aligned}
$$

Then, the energy balance equality (Lemma 2.6) implies

$$
\begin{aligned}
\left\|\left(\boldsymbol{E}_{1}-\boldsymbol{E}_{2}, \boldsymbol{H}_{1}-\boldsymbol{H}_{2}\right)(t)\right\|_{\boldsymbol{X}}^{2}=2 \int_{0}^{t}\left(\boldsymbol{u}_{1}(s)-\boldsymbol{u}_{2}(s), \boldsymbol{E}_{1}(s)-\boldsymbol{E}_{2}(s)\right)_{\boldsymbol{L}^{2}(\Omega)} \\
-\left(\varphi^{\gamma}\left(\cdot, \boldsymbol{E}_{1}(s)\right)-\varphi^{\gamma}\left(\cdot, \boldsymbol{E}_{2}(s)\right), \boldsymbol{E}_{1}(s)-\boldsymbol{E}_{2}(s)\right)_{\boldsymbol{L}^{2}(\Omega)} d s \quad \forall t \in[0, T] .
\end{aligned}
$$

Since $\left(\varphi^{\gamma}(\cdot, \boldsymbol{y})-\varphi^{\gamma}(\cdot, \boldsymbol{v}), \boldsymbol{y}-\boldsymbol{v}\right)_{\boldsymbol{L}^{2}(\Omega)} \geq 0$ holds for all $\boldsymbol{y}, \boldsymbol{v} \in \boldsymbol{L}^{2}(\Omega)$, we obtain that

$$
\begin{aligned}
\|\left(\boldsymbol{E}_{1}\right. & \left.-\boldsymbol{E}_{2}, \boldsymbol{H}_{1}-\boldsymbol{H}_{2}\right)(t)\left\|_{\boldsymbol{X}}^{2} \leq 2\right\| \boldsymbol{E}_{1}-\boldsymbol{E}_{2}\left\|_{\mathcal{C}\left([0, T], \boldsymbol{L}^{2}(\Omega)\right)} \int_{0}^{t}\right\| \boldsymbol{u}_{1}(s)-\boldsymbol{u}_{2}(s) \|_{\boldsymbol{L}^{2}(\Omega)} d s \\
& \leq 2 \underline{\epsilon}^{-1 / 2}\left\|\boldsymbol{E}_{1}-\boldsymbol{E}_{2}\right\|_{\mathcal{C}\left([0, T], \boldsymbol{L}_{\epsilon}^{2}(\Omega)\right)}\left\|\boldsymbol{u}_{1}-\boldsymbol{u}_{2}\right\|_{L^{1}\left((0, T), \boldsymbol{L}^{2}(\Omega)\right)} \\
& \leq 2 \underline{\epsilon}^{-1 / 2}\left\|\left(\boldsymbol{E}_{1}-\boldsymbol{E}_{2}, \boldsymbol{H}_{1}-\boldsymbol{H}_{2}\right)\right\|_{\mathcal{C}([0, T], \boldsymbol{X})}\left\|\boldsymbol{u}_{1}-\boldsymbol{u}_{2}\right\|_{L^{1}\left((0, T), \boldsymbol{L}^{2}(\Omega)\right)} \quad \forall t \in[0, T],
\end{aligned}
$$

from which the assertion follows.

Next, we consider $G_{\gamma}$ as an operator from $L^{2}\left((0, T), \boldsymbol{L}^{2}(\Omega)\right)$ to $L^{2}((0, T), \boldsymbol{X})$ :

$$
S_{\gamma}: L^{2}\left((0, T), \boldsymbol{L}^{2}(\Omega)\right) \rightarrow L^{2}((0, T), \boldsymbol{X}), \quad S_{\gamma}:=i G_{\gamma}
$$

where $i$ denotes the continuous injection $\mathcal{C}([0, T], \boldsymbol{X}) \hookrightarrow L^{2}([0, T], \boldsymbol{X})$. Our goal is to establish the weak Gâteaux-differentiability of $S_{\gamma}: L^{2}\left((0, T), \boldsymbol{L}^{2}(\Omega)\right) \rightarrow L^{2}((0, T), \boldsymbol{X})$. Let us note that for every fixed $x \in \Omega$, the function $\varphi^{\gamma}(x, \cdot): \mathbb{R}^{3} \rightarrow \mathbb{R}^{3}$ is infinitely differentiable. We denote the corresponding Jacobian matrix by

$$
\nabla_{y} \varphi^{\gamma}: \Omega \times \mathbb{R}^{3} \rightarrow \mathbb{R}^{3 \times 3}, \quad \nabla_{y} \varphi^{\gamma}(x, y)=\left(\frac{\partial \varphi_{i}^{\gamma}}{\partial y_{j}}(x, y)\right)_{1 \leq i, j \leq 3} .
$$

By straightforward computations,

$$
\nabla_{y} \varphi^{\gamma}(x, y)=\frac{j_{c}(x)}{\left(\gamma^{-2}+|y|^{2}\right)^{\frac{3}{2}}}\left(\begin{array}{ccc}
\gamma^{-2}+y_{2}^{2}+y_{3}^{2} & -y_{1} y_{2} & -y_{1} y_{3} \\
-y_{2} y_{1} & \gamma^{-2}+y_{1}^{2}+y_{3}^{2} & -y_{2} y_{3} \\
-y_{3} y_{1} & -y_{3} y_{2} & \gamma^{-2}+y_{1}^{2}+y_{2}^{2}
\end{array}\right)
$$

holds for all $(x, y) \in \Omega \times \mathbb{R}^{3}$. Hence, for all $(x, y) \in \Omega \times \mathbb{R}^{3}$, the Jacobian matrix $\nabla_{y} \varphi^{\gamma}(x, y) \in \mathbb{R}^{3 \times 3}$ is symmetric and positive semidefinite:

$$
\xi^{T} \nabla_{y} \varphi^{\gamma}(x, y) \xi \geq 0 \quad \text { for all }(x, y) \in \Omega \times \mathbb{R}^{3} \text { and all } \xi \in \mathbb{R}^{3} .
$$

Furthermore, there exists a constant $c>0$, depending only on $\gamma$ and $\mathfrak{j}_{c}$, such that

$$
\left|\nabla_{y} \varphi^{\gamma}(x, y)\right|_{2} \leq c \text { for all }(x, y) \in \Omega \times \mathbb{R}^{3},
$$

where $|\cdot|_{2}: \mathbb{R}^{3 \times 3} \rightarrow \mathbb{R}$ denotes the spectral norm on $\mathbb{R}^{3 \times 3}$.

Copyright ( by SIAM. Unauthorized reproduction of this article is prohibited. 
Lemma 4.13. The operator $S_{\gamma}: L^{2}\left((0, T), \boldsymbol{L}^{2}(\Omega)\right) \rightarrow L^{2}((0, T), \boldsymbol{X})$ is weakly directional differentiable. The weak directional derivative of $S_{\gamma}$ at $\boldsymbol{u} \in L^{2}\left((0, T), \boldsymbol{L}^{2}(\Omega)\right)$ in the direction $\delta \boldsymbol{u} \in L^{2}\left((0, T), \boldsymbol{L}^{2}(\Omega)\right)$ is given by $S_{\gamma}^{\prime}(\boldsymbol{u}) \delta \boldsymbol{u}=(\delta \boldsymbol{E}, \delta \boldsymbol{H})$, where $(\delta \boldsymbol{E}, \delta \boldsymbol{H}) \in \mathcal{C}([0, T], \boldsymbol{X})$ satisfies the following integral equation:

$$
(\delta \boldsymbol{E}, \delta \boldsymbol{H})(t)=\int_{0}^{t} \mathbb{T}_{t-s}\left(\epsilon^{-1}\left(\delta \boldsymbol{u}(s)-\nabla_{y} \varphi^{\gamma}(\cdot, \boldsymbol{E}(s)) \delta \boldsymbol{E}(s)\right), 0\right) d s \forall t \in[0, T]
$$

with $(\boldsymbol{E}, \boldsymbol{H})=G_{\gamma}(\boldsymbol{u})$.

Remark 4.14. In view of [3] (cf. Remark 4.2), $(\delta \boldsymbol{E}, \delta \boldsymbol{H})$ satisfies the weak formulation for the linearized equations of (4.1):

$$
\left\{\begin{array}{l}
\frac{d}{d t} \int_{\Omega} \epsilon \delta \boldsymbol{E}(t) \cdot \boldsymbol{v} d x-\int_{\Omega} \delta \boldsymbol{H}(t) \cdot \operatorname{curl} \boldsymbol{v} d x+\int_{\Omega} \nabla_{y} \varphi^{\gamma}(\cdot, \boldsymbol{E}(t)) \delta \boldsymbol{E}(t) \cdot \boldsymbol{v} d x \\
\frac{d}{d t} \int_{\Omega} \mu \delta \boldsymbol{H}(t) \cdot \boldsymbol{w} d x+\int_{\Omega} \delta \boldsymbol{E}(t) \cdot \operatorname{curl} \boldsymbol{w} d x=0, \\
\text { for a.e. } t \in(0, T) \text { and all }(\boldsymbol{v}, \boldsymbol{w}) \in \boldsymbol{H}_{0}(\operatorname{curl}) \times \boldsymbol{H}(t) \cdot \boldsymbol{v} d x, \\
(\boldsymbol{E}, \boldsymbol{H})(0)=\left(\boldsymbol{E}_{0}, \boldsymbol{H}_{0}\right) .
\end{array}\right.
$$

Proof. Let $\boldsymbol{u}, \delta \boldsymbol{u} \in L^{2}\left((0, T), \boldsymbol{L}^{2}(\Omega)\right)$ and $(\boldsymbol{E}, \boldsymbol{H})=G_{\gamma}(\boldsymbol{u})$. Further, for every $\tau \in \mathbb{R}^{+}$, we set $\left(\boldsymbol{E}_{\tau}, \boldsymbol{H}_{\tau}\right)=G_{\gamma}(\boldsymbol{u}+\tau \delta \boldsymbol{u})$. By definition, we have that

$$
\begin{aligned}
& \left(\frac{\boldsymbol{E}_{\tau}-\boldsymbol{E}}{\tau}, \frac{\boldsymbol{H}_{\tau}-\boldsymbol{H}}{\tau}\right)(t) \\
& \quad=\int_{0}^{t} \mathbb{T}_{t-s}\left(\epsilon^{-1}\left(\delta \boldsymbol{u}(s)-\frac{\varphi^{\gamma}\left(\cdot, \boldsymbol{E}_{\tau}(s)\right)-\varphi^{\gamma}(\cdot, \boldsymbol{E}(s))}{\tau}\right), 0\right) d s \quad \forall t \in[0, T] .
\end{aligned}
$$

Lemma 4.12 implies that $\left\{\left(\frac{\boldsymbol{E}_{\tau}-\boldsymbol{E}}{\tau}, \frac{\boldsymbol{H}_{\tau}-\boldsymbol{H}}{\tau}\right)\right\}_{\tau>0}$ is bounded in $L^{2}((0, T), \boldsymbol{X})$. For this reason, there exists a subsequence of $\left\{\left(\frac{\boldsymbol{E}_{\tau}-\boldsymbol{E}}{\tau}, \frac{\boldsymbol{H}_{\tau}-\boldsymbol{H}}{\tau}\right)\right\}_{\tau>0}$, which we denote without loss of generality again by the sequence itself, such that

$$
\left(\frac{\boldsymbol{E}_{\tau}-\boldsymbol{E}}{\tau}, \frac{\boldsymbol{H}_{\tau}-\boldsymbol{H}}{\tau}\right) \rightarrow(\delta \boldsymbol{E}, \delta \boldsymbol{H}) \quad \text { weakly in } L^{2}((0, T), \boldsymbol{X}) \text { as } \tau \rightarrow 0
$$

for some $(\delta \boldsymbol{E}, \delta \boldsymbol{H}) \in L^{2}((0, T), \boldsymbol{X})$. By the mean value theorem in integral form, it holds for almost all $(x, t) \in \Omega \times(0, T)$ that

$$
\begin{aligned}
& \frac{\varphi^{\gamma}\left(x, \boldsymbol{E}_{\tau}(x, t)\right)-\varphi^{\gamma}(x, \boldsymbol{E}(x, t))}{\tau} \\
& =\left(\int_{0}^{1} \nabla_{y} \varphi^{\gamma}\left(x, \boldsymbol{E}(x, t)+\vartheta\left(\boldsymbol{E}_{\tau}(x, t)-\boldsymbol{E}(x, t)\right)\right) d \vartheta\right) \frac{\boldsymbol{E}_{\tau}(x, t)-\boldsymbol{E}(x, t)}{\tau} \\
& =\nabla_{y} \varphi^{\gamma}(x, \boldsymbol{E}(x, t)) \frac{\boldsymbol{E}_{\tau}(x, t)-\boldsymbol{E}(x, t)}{\tau}+\boldsymbol{R}_{\tau}(x, t) \frac{\boldsymbol{E}_{\tau}(x, t)-\boldsymbol{E}(x, t)}{\tau}
\end{aligned}
$$

with $\boldsymbol{R}_{\tau}(x, t)=\left(\int_{0}^{1} \nabla_{y} \varphi^{\gamma}\left(x, \boldsymbol{E}(x, t)+\vartheta\left(\boldsymbol{E}_{\tau}(x, t)-\boldsymbol{E}(x, t)\right)\right) d \vartheta-\nabla_{y} \varphi^{\gamma}(x, \boldsymbol{E}(x, t))\right)$. By virtue of Lemma 4.12,

$$
\boldsymbol{E}_{\tau} \rightarrow \boldsymbol{E} \quad \text { strongly in } \mathcal{C}\left([0, T], \boldsymbol{L}^{2}(\Omega)\right) \text { as } \tau \rightarrow 0 .
$$


For this reason and making use of the boundedness property (4.34), Lebesgue's dominated convergence theorem implies for every $\boldsymbol{v} \in L^{2}\left((0, T), \boldsymbol{L}^{2}(\Omega)\right)$ that

$$
\boldsymbol{R}_{\tau} \boldsymbol{v} \rightarrow 0 \text { strongly in } L^{2}\left((0, T), \boldsymbol{L}^{2}(\Omega)\right) \text { as } \tau \rightarrow 0 .
$$

Now, according to (4.38), it holds for every $\boldsymbol{v} \in L^{2}\left((0, T), \boldsymbol{L}^{2}(\Omega)\right)$ that

$$
\begin{aligned}
& \int_{0}^{T} \int_{\Omega} \frac{\varphi^{\gamma}\left(x, \boldsymbol{E}_{\tau}(x, t)\right)-\varphi^{\gamma}(x, \boldsymbol{E}(x, t))}{\tau} \cdot \boldsymbol{v}(x, t) d x d t \\
& =\int_{0}^{T} \int_{\Omega} \frac{\boldsymbol{E}_{\tau}(x, t)-\boldsymbol{E}(x, t)}{\tau} \cdot \nabla_{y} \varphi^{\gamma}(x, \boldsymbol{E}(x, t)) \boldsymbol{v}(x, t) \\
& \quad+\frac{\boldsymbol{E}_{\tau}(x, t)-\boldsymbol{E}(x, t)}{\tau} \cdot \boldsymbol{R}_{\tau}(x, t) \boldsymbol{v}(x, t) d x d t,
\end{aligned}
$$

since for all $(x, y) \in \Omega \times \mathbb{R}^{3}$ the Jacobian matrix $\nabla_{y} \varphi^{\gamma}(x, y) \in \mathbb{R}^{3 \times 3}$ is symmetric. Consequently, (4.37) and (4.39) imply

$$
\begin{aligned}
& \int_{0}^{T} \int_{\Omega} \frac{\varphi^{\gamma}\left(x, \boldsymbol{E}_{\tau}(x, t)\right)-\varphi^{\gamma}(x, \boldsymbol{E}(x, t))}{\tau} \cdot \boldsymbol{v}(x, t) d x d t \\
& \rightarrow \int_{0}^{T} \int_{\Omega} \delta \boldsymbol{E}(x, t) \cdot \nabla_{y} \varphi^{\gamma}(x, \boldsymbol{E}(x, t)) \boldsymbol{v}(x, t) d x d t \quad \text { as } \tau \rightarrow 0 \quad \forall \boldsymbol{v} \in L^{2}\left((0, T), \boldsymbol{L}^{2}(\Omega)\right) .
\end{aligned}
$$

In other words,

$$
\frac{\varphi^{\gamma}\left(\cdot, \boldsymbol{E}_{\tau}\right)-\varphi^{\gamma}(\cdot, \boldsymbol{E})}{\tau} \rightarrow \nabla_{y} \varphi^{\gamma}(\cdot, \boldsymbol{E}) \delta \boldsymbol{E} \quad \text { weakly in } L^{2}\left((0, T), \boldsymbol{L}^{2}(\Omega)\right) \text { as } \tau \rightarrow 0 .
$$

This weak convergence applied to (4.36) yields that the weak limit $(\delta \boldsymbol{E}, \delta \boldsymbol{H})$ of (4.37) satisfies

$$
(\delta \boldsymbol{E}, \delta \boldsymbol{H})(t)=\int_{0}^{t} \mathbb{T}_{t-s}\left(\epsilon^{-1}\left(\delta \boldsymbol{u}(s)-\nabla_{y} \varphi^{\gamma}(\cdot, \boldsymbol{E}(s)) \delta \boldsymbol{E}(s)\right), 0\right) d s \forall t \in[0, T]
$$

Now, the assertion is true if the integral equation (4.40) admits a unique solution. Suppose that $(\widetilde{\delta \boldsymbol{E}}, \widetilde{\delta \boldsymbol{H}}) \in \mathcal{C}([0, T], \boldsymbol{X})$ is another solution of (4.40). Then,

$$
(\delta \boldsymbol{E}-\widetilde{\delta \boldsymbol{E}}, \delta \boldsymbol{H}-\widetilde{\delta \boldsymbol{H}})(t)=\int_{0}^{t} \mathbb{T}_{t-s}\left(\epsilon^{-1}\left(-\nabla_{y} \varphi^{\gamma}(\cdot, \boldsymbol{E}(s))(\delta \boldsymbol{E}(s)-\widetilde{\delta \boldsymbol{E}}(s))\right), 0\right) d s
$$

for all $t \in[0, T]$. Consequently, Lemma 2.6 and (4.33) imply

$$
\begin{aligned}
& \|(\delta \boldsymbol{E}-\widetilde{\delta \boldsymbol{E}}, \delta \boldsymbol{H}-\widetilde{\delta \boldsymbol{H}})(t)\|_{\boldsymbol{X}}^{2} \\
& =-2 \int_{0}^{t}\left(\nabla_{y} \varphi^{\gamma}(\cdot, \boldsymbol{E}(s))(\delta \boldsymbol{E}(s)-\widetilde{\delta \boldsymbol{E}}(s)), \delta \boldsymbol{E}(s)-\widetilde{\delta \boldsymbol{E}}(s)\right)_{\boldsymbol{L}^{2}(\Omega)} d s \leq 0 \quad \forall t \in[0, T] .
\end{aligned}
$$

This completes the proof.

Corollary 4.15. The operator $S_{\gamma}: L^{2}\left((0, T), \boldsymbol{L}^{2}(\Omega)\right) \rightarrow L^{2}((0, T), \boldsymbol{X})$ is weakly Gâteaux-differentiable.

Proof. Let $\boldsymbol{u} \in L^{2}\left((0, T), \boldsymbol{L}^{2}(\Omega)\right)$ and $(\boldsymbol{E}, \boldsymbol{H})=G_{\gamma}(\boldsymbol{u})$. From (4.35), it is obvious that the mapping $S_{\gamma}^{\prime}(\boldsymbol{u}): L^{2}\left((0, T), \boldsymbol{L}^{2}(\Omega)\right) \rightarrow L^{2}((0, T), \boldsymbol{X})$ is linear. Let now 
$\delta \boldsymbol{u} \in L^{2}\left((0, T), \boldsymbol{L}^{2}(\Omega)\right)$, and we set $S_{\gamma}^{\prime}(\boldsymbol{u}) \delta \boldsymbol{u}=(\delta \boldsymbol{E}, \delta \boldsymbol{H})$. The energy balance equality (Lemma 2.6) in (4.35) implies

$$
\|(\delta \boldsymbol{E}, \delta \boldsymbol{H})(t)\|_{\boldsymbol{X}}^{2}=2 \int_{0}^{t}(\delta \boldsymbol{u}(s), \delta \boldsymbol{E}(s))_{\boldsymbol{L}^{2}(\Omega)}-\left(\nabla_{y} \varphi^{\gamma}(\cdot, \boldsymbol{E}(s)) \delta \boldsymbol{E}(s), \delta \boldsymbol{E}(s)\right)_{\boldsymbol{L}^{2}(\Omega)} d s
$$

for all $t \in[0, T]$. It follows therefore from (4.33) that

$$
\|(\delta \boldsymbol{E}, \delta \boldsymbol{H})(t)\|_{\boldsymbol{X}}^{2} \leq 2 \int_{0}^{t}(\delta \boldsymbol{u}(s), \delta \boldsymbol{E}(s))_{\boldsymbol{L}^{2}(\Omega)} d s \quad \forall t \in[0, T],
$$

and so there exists a constant $c>0$, independent of $\delta \boldsymbol{u}$ and $(\delta \boldsymbol{E}, \delta \boldsymbol{H})$, such that $\|(\delta \boldsymbol{E}, \delta \boldsymbol{H})\|_{L^{2}((0, T), \boldsymbol{X})} \leq c\|\delta \boldsymbol{u}\|_{L^{2}\left((0, T), \boldsymbol{L}^{2}(\Omega)\right)}$,

In view of Corollary 4.15 , for every $\boldsymbol{u} \in L^{2}\left((0, T), \boldsymbol{L}^{2}(\Omega)\right)$, the (Hilbert-) adjoint operator $S_{\gamma}^{\prime}(\boldsymbol{u})^{*}: L^{2}((0, T), \boldsymbol{X}) \rightarrow L^{2}\left((0, T), \boldsymbol{L}^{2}(\Omega)\right)$ exists as a linear and bounded operator, which is defined by

$$
\left((\boldsymbol{e}, \boldsymbol{h}), S_{\gamma}^{\prime}(\boldsymbol{u}) \delta \boldsymbol{u}\right)_{L^{2}((0, T), \boldsymbol{X})}=\left(S_{\gamma}^{\prime}(\boldsymbol{u})^{*}(\boldsymbol{e}, \boldsymbol{h}), \delta \boldsymbol{u}\right)_{L^{2}\left((0, T), \boldsymbol{L}^{2}(\Omega)\right)}
$$

for all $\delta \boldsymbol{u} \in L^{2}\left((0, T), \boldsymbol{L}^{2}(\Omega)\right)$ and all $(\boldsymbol{e}, \boldsymbol{h}) \in L^{2}((0, T), \boldsymbol{X})$. By standard arguments (see the appendix; cf. [21]), the adjoint operator $S_{\gamma}^{\prime}(\boldsymbol{u})^{*}: L^{2}((0, T), \boldsymbol{X}) \rightarrow$ $L^{2}\left((0, T), \boldsymbol{L}^{2}(\Omega)\right)$ satisfies

(4.41a) $S_{\gamma}^{\prime}(\boldsymbol{u})^{*}(\boldsymbol{e}, \boldsymbol{h})=\boldsymbol{K}$,

$$
(\boldsymbol{K}, \boldsymbol{Q})(t)=\int_{t}^{T} \mathbb{T}_{t-s}\left(\boldsymbol{e}(s)-\epsilon^{-1} \nabla_{y} \varphi^{\gamma}(\cdot, \boldsymbol{E}(s)) \boldsymbol{K}(s), \boldsymbol{h}(s)\right) d s \quad \forall t \in[0, T]
$$

where $(\boldsymbol{E}, \boldsymbol{H})=G_{\gamma}(\boldsymbol{u}) \in \mathcal{C}([0, T], \boldsymbol{X})$. Note that a classical contraction argument (cf. [16, Theorem 1.2, p. 184]) implies that the integral equation (4.41b) admits for every $(\boldsymbol{e}, \boldsymbol{h}) \in L^{2}((0, T), \boldsymbol{X})$ a unique solution $(\boldsymbol{K}, \boldsymbol{Q}) \in \mathcal{C}([0, T], \boldsymbol{X})$.

Let us now introduce the objective functional $f_{\gamma}: H^{2}\left((0, T), \boldsymbol{L}^{2}(\Omega)\right) \rightarrow \mathbb{R}$,

$$
\begin{array}{r}
f_{\gamma}(\boldsymbol{u}):=\frac{1}{2}\left\|S_{\gamma}(\boldsymbol{u})-\left(\boldsymbol{E}_{d}, \boldsymbol{H}_{d}\right)\right\|_{L^{2}\left((0, T), \boldsymbol{L}^{2}(\Omega) \times \boldsymbol{L}^{2}(\Omega)\right)}^{2}+\frac{\kappa}{2}\|\boldsymbol{u}\|_{H^{2}\left((0, T), \boldsymbol{L}^{2}(\Omega)\right)}^{2} \\
+\frac{1}{2}\|\boldsymbol{u}-\overline{\boldsymbol{u}}\|_{H^{2}\left((0, T), \boldsymbol{L}^{2}(\Omega)\right)}^{2} .
\end{array}
$$

We see that $\left(\mathrm{P}_{\gamma}^{\bar{u}}\right)$ can be equivalently expressed as the following optimization problem in Hilbert space:

$$
\min _{\boldsymbol{u} \in H^{2}((0, T), \boldsymbol{H}(\operatorname{div}=0))} f_{\gamma}(\boldsymbol{u})
$$

Corollary 4.16. For every $\gamma>0$, the functional $f_{\gamma}: H^{2}\left((0, T), \boldsymbol{L}^{2}(\Omega)\right) \rightarrow \mathbb{R}$ is Gâteaux-differentiable with the Gâteaux-derivative:

$$
\begin{aligned}
f_{\gamma}^{\prime}(\boldsymbol{u}) \delta \boldsymbol{u}= & \left(S_{\gamma}(\boldsymbol{u})-\left(\boldsymbol{E}_{d}, \boldsymbol{H}_{d}\right), S_{\gamma}^{\prime}(\boldsymbol{u}) \delta \boldsymbol{u}\right)_{L^{2}\left((0, T), \boldsymbol{L}^{2}(\Omega) \times \boldsymbol{L}^{2}(\Omega)\right)} \\
& +(\kappa \boldsymbol{u}+\boldsymbol{u}-\overline{\boldsymbol{u}}, \delta \boldsymbol{u})_{H^{2}\left((0, T), \boldsymbol{L}^{2}(\Omega)\right)}
\end{aligned}
$$

for all $\boldsymbol{u}, \delta \boldsymbol{u} \in H^{2}\left((0, T), \boldsymbol{L}^{2}(\Omega)\right)$.

Proof. By classical arguments, the assertion follows from Corollary 4.15.

Copyright ( by SIAM. Unauthorized reproduction of this article is prohibited. 
TheOREM 4.17. Let $\gamma>0$ and $\left(\overline{\boldsymbol{E}}^{\gamma}, \overline{\boldsymbol{H}}^{\gamma}, \overline{\boldsymbol{u}}^{\gamma}\right) \in \mathcal{C}([0, T], D(\mathcal{A})) \cap \mathcal{C}^{1}([0, T], \boldsymbol{X}) \times$ $H^{2}((0, T), \boldsymbol{H}(\operatorname{div}=0))$ denote an optimal solution of $\left(\mathrm{P}_{\gamma}^{\bar{u}}\right)$. Then, there exists $\left(\overline{\boldsymbol{K}}^{\gamma}, \overline{\boldsymbol{Q}}^{\gamma}\right)$ $\in \mathcal{C}([0, T], \boldsymbol{X})$ satisfying

$$
\begin{gathered}
\left(\overline{\boldsymbol{K}}^{\gamma}, \overline{\boldsymbol{Q}}^{\gamma}\right)(t)=\int_{t}^{T} \mathbb{T}_{t-s}\left(\epsilon^{-1}\left(\overline{\boldsymbol{E}}^{\gamma}(s)-\boldsymbol{E}_{d}(s)-\nabla_{y} \varphi^{\gamma}\left(\cdot, \overline{\boldsymbol{E}}^{\gamma}(s)\right) \overline{\boldsymbol{K}}^{\gamma}(s)\right),\right. \\
\left.\mu^{-1}\left(\overline{\boldsymbol{H}}^{\gamma}(s)-\boldsymbol{H}_{d}(s)\right)\right) d s \quad \forall t \in[0, T], \\
\left(\kappa \overline{\boldsymbol{u}}^{\gamma}+\overline{\boldsymbol{u}}^{\gamma}-\overline{\boldsymbol{u}}, \delta \boldsymbol{u}\right)_{H^{2}\left((0, T), \boldsymbol{L}^{2}(\Omega)\right)}=-\left(\overline{\boldsymbol{K}}^{\gamma}, \delta \boldsymbol{u}\right)_{L^{2}\left((0, T), \boldsymbol{L}^{2}(\Omega)\right)} \\
\forall \delta \boldsymbol{u} \in H^{2}((0, T), \boldsymbol{H}(\operatorname{div}=0)) .
\end{gathered}
$$

Proof. The necessary optimality condition for $\left(\mathrm{P}_{\gamma}^{\bar{u}}\right)$ reads as

$$
f_{\gamma}^{\prime}\left(\overline{\boldsymbol{u}}^{\gamma}\right) \delta \boldsymbol{u}=0 \quad \forall \delta \boldsymbol{u} \in H^{2}((0, T), \boldsymbol{H}(\operatorname{div}=0)),
$$

which is according to (4.43) equivalent to

$$
\begin{aligned}
& \left(\left(\epsilon^{-1}\left(\overline{\boldsymbol{E}}^{\gamma}-\boldsymbol{E}_{d}\right), \mu^{-1}\left(\overline{\boldsymbol{H}}^{\gamma}-\boldsymbol{H}_{d}\right)\right), S_{\gamma}^{\prime}\left(\overline{\boldsymbol{u}}^{\gamma}\right) \delta \boldsymbol{u}\right)_{L^{2}((0, T), \boldsymbol{X})} \\
& +\left(\kappa \overline{\boldsymbol{u}}^{\gamma}+\overline{\boldsymbol{u}}^{\gamma}-\overline{\boldsymbol{u}}, \delta \boldsymbol{u}\right)_{H^{2}\left((0, T), \boldsymbol{L}^{2}(\Omega)\right)}=0 \quad \forall \delta \boldsymbol{u} \in H^{2}((0, T), \boldsymbol{H}(\operatorname{div}=0)) .
\end{aligned}
$$

Thus, by virtue of (4.41), we conclude that (4.44) is valid.

5. Optimality system for (P). A standard strategy to derive an optimality system for $(\mathrm{P})$ would be based on the boundedness of the sequence

$$
\left\{\nabla_{y} \varphi^{\gamma}\left(\cdot, \overline{\boldsymbol{E}}^{\gamma}\right) \overline{\boldsymbol{K}}^{\gamma}\right\}_{\gamma>0}
$$

in the dual space of some proper Hilbert space. This approach is well-known for the optimal control of elliptic variational equalities (see, e.g., [4]). In our case, however, the boundedness of (5.1) cannot be expected due to lack of regularity properties in the (regularized) adjoint state $\left(\overline{\boldsymbol{K}}^{\gamma}, \overline{\boldsymbol{Q}}^{\gamma}\right)$. Our strategy to derive an optimality system for (P) is based on the fact that the sequence $\left\{\left(\overline{\boldsymbol{\lambda}}^{\boldsymbol{\gamma}}, \overline{\boldsymbol{\eta}}^{\boldsymbol{\gamma}}\right)\right\}_{\gamma>0}$, defined by

$$
\left(\overline{\boldsymbol{\lambda}}^{\gamma}, \overline{\boldsymbol{\eta}}^{\gamma}\right)(t):=-\int_{t}^{T} \mathbb{T}_{t-s}\left(\epsilon^{-1} \nabla_{y} \varphi^{\gamma}\left(\cdot, \overline{\boldsymbol{E}}^{\gamma}(s)\right) \overline{\boldsymbol{K}}^{\gamma}(s), 0\right) d s \quad \forall t \in[0, T]
$$

is bounded in $\mathcal{C}([0, T], \boldsymbol{X})$.

Lemma 5.1. For every $\gamma>0$, let $\left(\overline{\boldsymbol{E}}^{\gamma}, \overline{\boldsymbol{H}}^{\gamma}, \overline{\boldsymbol{u}}^{\gamma}\right) \in \mathcal{C}([0, T], D(\mathcal{A})) \cap \mathcal{C}^{1}([0, T], \boldsymbol{X}) \times$ $H^{2}((0, T), \boldsymbol{H}(\operatorname{div}=0))$ denote an optimal solution of $\left(\mathrm{P}_{\gamma}^{\bar{u}}\right)$. Furthermore, for every $\gamma>0$, let $\left(\overline{\boldsymbol{K}}^{\gamma}, \overline{\boldsymbol{Q}}^{\gamma}\right),\left(\overline{\boldsymbol{\lambda}}^{\gamma}, \overline{\boldsymbol{\eta}}^{\gamma}\right) \in \mathcal{C}([0, T], \boldsymbol{X})$ be as in Theorem 4.17 and (5.2). Then, the sequences $\left\{\left(\overline{\boldsymbol{K}}^{\gamma}, \overline{\boldsymbol{Q}}^{\gamma}\right)\right\}_{\gamma>0}$ and $\left\{\left(\overline{\boldsymbol{\lambda}}^{\gamma}, \overline{\boldsymbol{\eta}}^{\gamma}\right)\right\}_{\gamma>0}$ are bounded in $\mathcal{C}([0, T], \boldsymbol{X})$.

Proof. Applying the time-transformation $\tau=T-t$ and $\sigma=T-s$ in (4.44a) yields

$$
\begin{aligned}
\left(\overline{\boldsymbol{K}}^{\gamma}, \overline{\boldsymbol{Q}}^{\gamma}\right)(T-\tau) \\
=-\int_{\tau}^{0} \mathbb{T}_{\sigma-\tau}\left(\epsilon ^ { - 1 } \left(\overline{\boldsymbol{E}}^{\gamma}(T-\sigma)-\boldsymbol{E}_{d}(T-\sigma)\right.\right. \\
\left.\left.\quad-\nabla_{y} \varphi^{\gamma}\left(\cdot, \overline{\boldsymbol{E}}^{\gamma}(T-\sigma)\right) \overline{\boldsymbol{K}}^{\gamma}(T-\sigma)\right), \mu^{-1}\left(\overline{\boldsymbol{H}}^{\gamma}(T-\sigma)-\boldsymbol{H}_{d}(T-\sigma)\right)\right) d \sigma
\end{aligned}
$$

Copyright $(\odot$ by SIAM. Unauthorized reproduction of this article is prohibited. 


$$
\begin{aligned}
= & \int_{0}^{\tau} \mathbb{T}_{\tau-\sigma}^{*}\left(\epsilon ^ { - 1 } \left(\overline{\boldsymbol{E}}^{\gamma}(T-\sigma)-\boldsymbol{E}_{d}(T-\sigma)\right.\right. \\
& \left.\left.-\nabla_{y} \varphi^{\gamma}\left(\cdot, \overline{\boldsymbol{E}}^{\gamma}(T-\sigma)\right) \overline{\boldsymbol{K}}^{\gamma}(T-\sigma)\right), \mu^{-1}\left(\overline{\boldsymbol{H}}^{\gamma}(T-\sigma)-\boldsymbol{H}_{d}(T-\sigma)\right)\right) d \sigma,
\end{aligned}
$$

since $\left\{\mathbb{T}_{t}\right\}_{t \in \mathbb{R}}$ is unitary. Then, the energy balance equality (Lemma 2.6) implies

$$
\begin{aligned}
& \left\|\left(\overline{\boldsymbol{K}}^{\gamma}, \overline{\boldsymbol{Q}}^{\gamma}\right)(T-\tau)\right\|_{\boldsymbol{X}}^{2} \\
& =2 \int_{0}^{\tau}\left(\overline{\boldsymbol{E}}^{\gamma}(T-\sigma)-\boldsymbol{E}_{d}(T-\sigma)-\nabla_{y} \varphi^{\gamma}\left(\cdot, \overline{\boldsymbol{E}}^{\gamma}(T-\sigma)\right) \overline{\boldsymbol{K}}^{\gamma}(T-\sigma), \overline{\boldsymbol{K}}^{\gamma}(T-\sigma)\right)_{\boldsymbol{L}^{2}(\Omega)} \\
& \quad+\left(\overline{\boldsymbol{H}}^{\gamma}(T-\sigma)-\boldsymbol{H}_{d}(T-\sigma), \overline{\boldsymbol{Q}}^{\gamma}(T-\sigma)\right)_{\boldsymbol{L}^{2}(\Omega)} d \sigma \\
& \underbrace{\leq}_{\left(\mathrm{A}^{4.33)}\right.} 2 \int_{0}^{\tau}\left(\overline{\boldsymbol{E}}^{\gamma}(T-\sigma)-\boldsymbol{E}_{d}(T-\sigma), \overline{\boldsymbol{K}}^{\gamma}(T-\sigma)\right)_{\boldsymbol{L}^{2}(\Omega)} \\
& \left.\quad+(T-\sigma)-\boldsymbol{H}_{d}(T-\sigma), \overline{\boldsymbol{Q}}^{\gamma}(T-\sigma)\right)_{\boldsymbol{L}^{2}(\Omega)} d \sigma \quad \forall \tau \in[0, T] .
\end{aligned}
$$

It follows therefore from the boundedness of $\left\{\left(\overline{\boldsymbol{E}}^{\gamma}, \overline{\boldsymbol{H}}^{\gamma}\right)\right\}_{\gamma>0} \subset \mathcal{C}([0, T], D(\mathcal{A})) \cap$ $\mathcal{C}^{1}([0, T], \boldsymbol{X})$ (Lemma 4.7) that the sequence $\left\{\left(\overline{\boldsymbol{K}}^{\gamma}, \overline{\boldsymbol{Q}}^{\gamma}\right)\right\}_{\gamma>0} \subset \mathcal{C}([0, T], \boldsymbol{X})$ is bounded. Now, according to (4.44a) and (5.2), we have that

$$
\left(\overline{\boldsymbol{K}}^{\gamma}, \overline{\boldsymbol{Q}}^{\gamma}\right)(t)=\int_{t}^{T} \mathbb{T}_{t-s}\left(\epsilon^{-1}\left(\overline{\boldsymbol{E}}^{\gamma}(s)-\boldsymbol{E}_{d}(s)\right), \mu^{-1}\left(\overline{\boldsymbol{H}}^{\gamma}(s)-\boldsymbol{H}_{d}(s)\right)\right) d s+\left(\overline{\boldsymbol{\lambda}}^{\gamma}, \overline{\boldsymbol{\eta}}^{\gamma}\right)(t)
$$

for all $t \in[0, T]$. Thus, in view of the boundedness of $\left\{\left(\overline{\boldsymbol{K}}^{\gamma}, \overline{\boldsymbol{Q}}^{\gamma}\right)\right\}_{\gamma>0} \subset \mathcal{C}([0, T], \boldsymbol{X})$ and Lemma 4.7 , we come to the conclusion that $\left\{\left(\overline{\boldsymbol{\lambda}}^{\gamma}, \overline{\boldsymbol{\eta}}^{\gamma}\right)\right\}_{\gamma>0} \subset \mathcal{C}([0, T], \boldsymbol{X})$ is bounded.

Note that the inequality derived in the above proof implies that

$$
\begin{aligned}
\left\|\left(\overline{\boldsymbol{K}}^{\gamma}, \overline{\boldsymbol{Q}}^{\gamma}\right)\right\|_{L^{\infty}((0, T), \boldsymbol{X})} & \leq 2 \underline{\epsilon}^{-1 / 2}\left\|\overline{\boldsymbol{E}}^{\gamma}-\boldsymbol{E}_{d}\right\|_{L^{1}\left((0, T), \boldsymbol{L}^{2}(\Omega)\right)} \\
& +2 \underline{\mu}^{-1 / 2}\left\|\overline{\boldsymbol{H}}^{\gamma}-\boldsymbol{H}_{d}\right\|_{L^{1}\left((0, T), \boldsymbol{L}^{2}(\Omega)\right)} \quad \forall \gamma>0 .
\end{aligned}
$$

Finally, making use of Theorem 4.11, Theorem 4.17, and Lemma 5.1, we are able to prove the following necessary optimality conditions for $(\mathrm{P})$.

TheOREM 5.2. Let $(\overline{\boldsymbol{E}}, \overline{\boldsymbol{H}}, \overline{\boldsymbol{u}}) \in \mathcal{F}_{\text {feas }}$ be an optimal solution of $(\mathrm{P})$ according to Defintion 3.3. Furthermore, let $\overline{\boldsymbol{J}} \in L^{2}\left((0, T), \boldsymbol{L}^{2}(\Omega)\right)$ denote the associated optimal current density. Then, there exist $(\overline{\boldsymbol{K}}, \overline{\boldsymbol{Q}}),(\overline{\boldsymbol{\lambda}}, \overline{\boldsymbol{\eta}}) \in L^{\infty}((0, T), \boldsymbol{X})$ such that

$$
\begin{aligned}
& (\overline{\boldsymbol{E}}, \overline{\boldsymbol{H}})(t)=\mathbb{T}_{t}\left(\boldsymbol{E}_{0}, \boldsymbol{H}_{0}\right)+\int_{0}^{t} \mathbb{T}_{t-s}\left(\epsilon^{-1}(\overline{\boldsymbol{u}}(s)-\overline{\boldsymbol{J}}(s)), 0\right) d s \quad \forall t \in[0, T], \\
& \overline{\boldsymbol{J}}(x, t) \cdot \overline{\boldsymbol{E}}(x, t)=j_{c}(x)|\overline{\boldsymbol{E}}(x, t)| \quad \text { for a.e. }(x, t) \in \Omega \times(0, T), \\
& |\overline{\boldsymbol{J}}(x, t)| \leq j_{c}(x) \quad \text { for a.e. }(x, t) \in \Omega \times(0, T), \\
& (\overline{\boldsymbol{K}}, \overline{\boldsymbol{Q}})(t)=\int_{t}^{T} \mathbb{T}_{t-s}\left(\epsilon^{-1}\left(\overline{\boldsymbol{E}}(s)-\boldsymbol{E}_{d}(s)\right), \mu^{-1}\left(\overline{\boldsymbol{H}}(s)-\boldsymbol{H}_{d}(s)\right)\right) d s+(\overline{\boldsymbol{\lambda}}, \overline{\boldsymbol{\eta}})(t) \\
& \quad \text { for a.e. } t \in(0, T), \\
& (\overline{\boldsymbol{u}}, \delta \boldsymbol{u})_{H^{2}\left((0, T), \boldsymbol{L}^{2}(\Omega)\right)}=-\kappa^{-1}(\overline{\boldsymbol{K}}, \delta \boldsymbol{u})_{L^{2}\left((0, T), \boldsymbol{L}^{2}(\Omega)\right)} \quad \forall \delta \boldsymbol{u} \in H^{2}((0, T), \boldsymbol{H}(\operatorname{div}=0)),
\end{aligned}
$$




$$
\begin{gathered}
\|(\overline{\boldsymbol{\lambda}}, \overline{\boldsymbol{\eta}})\|_{L^{\infty}((0, T), \boldsymbol{X})} \leq\left\|\left(\epsilon^{-1}\left(\overline{\boldsymbol{E}}-\boldsymbol{E}_{d}\right), \mu^{-1}\left(\overline{\boldsymbol{H}}-\boldsymbol{H}_{d}\right)\right)\right\|_{L^{1}((0, T), \boldsymbol{X})} \\
+\|(\overline{\boldsymbol{K}}, \overline{\boldsymbol{Q}})\|_{L^{\infty}((0, T), \boldsymbol{X})} \\
\begin{array}{c}
\|(\overline{\boldsymbol{K}}, \overline{\boldsymbol{Q}})\|_{L^{\infty}((0, T), \boldsymbol{X}) \leq} \leq \sqrt{8 T} \max \left\{\underline{\epsilon}^{-1 / 2}, \underline{\mu}^{-1 / 2}\right\}\left(\left\|\overline{\boldsymbol{E}}-\boldsymbol{E}_{d}\right\|_{L^{2}\left((0, T), \boldsymbol{L}^{2}(\Omega)\right)}^{2}\right. \\
\left.+\left\|\overline{\boldsymbol{H}}-\boldsymbol{H}_{d}\right\|_{L^{2}\left((0, T), L^{2}(\Omega)\right)}^{2}\right)^{1 / 2}
\end{array}
\end{gathered}
$$

Proof. By Lemma 5.1, there exist subsequences of $\left\{\left(\overline{\boldsymbol{K}}^{\gamma}, \overline{\boldsymbol{Q}}^{\gamma}\right)\right\}_{\gamma>0}$ and $\left\{\left(\overline{\boldsymbol{\lambda}}^{\boldsymbol{\gamma}}, \overline{\boldsymbol{\eta}}^{\boldsymbol{\gamma}}\right)\right\}_{\gamma>0}$, which we denote in the same way, such that

$$
\begin{cases}\left(\overline{\boldsymbol{K}}^{\gamma}, \overline{\boldsymbol{Q}}^{\gamma}\right)-(\overline{\boldsymbol{K}}, \overline{\boldsymbol{Q}}) & \text { weakly star in } L^{\infty}((0, T), \boldsymbol{X}) \text { as } \gamma \rightarrow \infty, \\ \left(\overline{\boldsymbol{\lambda}}^{\gamma}, \overline{\boldsymbol{\eta}}^{\gamma}\right)-(\overline{\boldsymbol{\lambda}}, \overline{\boldsymbol{\eta}}) & \text { weakly star in } L^{\infty}((0, T), \boldsymbol{X}) \text { as } \gamma \rightarrow \infty,\end{cases}
$$

for some $(\overline{\boldsymbol{K}}, \overline{\boldsymbol{Q}}),(\overline{\boldsymbol{\lambda}}, \overline{\boldsymbol{\eta}}) \in L^{\infty}((0, T), \boldsymbol{X})$. On the other hand, according to (5.3), we have that

$$
\left(\overline{\boldsymbol{K}}^{\gamma}, \overline{\boldsymbol{Q}}^{\gamma}\right)=\left(\overline{\boldsymbol{v}}^{\gamma}, \overline{\boldsymbol{w}}^{\gamma}\right)+\left(\overline{\boldsymbol{\lambda}}^{\gamma}, \overline{\boldsymbol{\eta}}^{\gamma}\right)
$$

where

$$
\left(\overline{\boldsymbol{v}}^{\gamma}, \overline{\boldsymbol{w}}^{\gamma}\right)(t):=\int_{t}^{T} \mathbb{T}_{t-s}\left(\epsilon^{-1}\left(\overline{\boldsymbol{E}}^{\gamma}(s)-\boldsymbol{E}_{d}(s)\right), \mu^{-1}\left(\overline{\boldsymbol{H}}^{\gamma}(s)-\boldsymbol{H}_{d}(s)\right)\right) d s \quad \forall t \in[0, T] .
$$

By virtue of Theorem 4.11, it holds that

$$
\left(\overline{\boldsymbol{v}}^{\gamma}, \overline{\boldsymbol{w}}^{\gamma}\right) \rightarrow(\overline{\boldsymbol{v}}, \overline{\boldsymbol{w}}) \quad \text { weakly in } L^{2}((0, T), \boldsymbol{X}) \text { as } \gamma \rightarrow \infty
$$

with

$$
(\overline{\boldsymbol{v}}, \overline{\boldsymbol{w}})(t)=\int_{t}^{T} \mathbb{T}_{t-s}\left(\epsilon^{-1}\left(\overline{\boldsymbol{E}}(s)-\boldsymbol{E}_{d}(s)\right), \mu^{-1}\left(\overline{\boldsymbol{H}}(s)-\boldsymbol{H}_{d}(s)\right)\right) d s \forall t \in[0, T] .
$$

In view of (5.5)-(5.8) along with (4.44b) and Theorem 4.11, we obtain that

$$
(\overline{\boldsymbol{K}}, \overline{\boldsymbol{Q}})(t)=\int_{t}^{T} \mathbb{T}_{t-s}\left(\epsilon^{-1}\left(\overline{\boldsymbol{E}}(s)-\boldsymbol{E}_{d}(s)\right), \mu^{-1}\left(\overline{\boldsymbol{H}}(s)-\boldsymbol{H}_{d}(s)\right)\right) d s+(\overline{\boldsymbol{\lambda}}, \overline{\boldsymbol{\eta}})(t)
$$

for a.e. $t \in(0, T)$, and

$$
(\overline{\boldsymbol{u}}, \delta \boldsymbol{u})_{H^{2}\left((0, T), \boldsymbol{L}^{2}(\Omega)\right)}=-\kappa^{-1}(\overline{\boldsymbol{K}}, \delta \boldsymbol{u})_{L^{2}\left((0, T), \boldsymbol{L}^{2}(\Omega)\right)} \quad \forall \delta \boldsymbol{u} \in H^{2}((0, T), \boldsymbol{H}(\operatorname{div}=0)) .
$$

Since $\{\mathbb{T}\}_{t \in \mathbb{R}}$ is unitary, the identity (5.9) immediately implies the following inequality:

$$
\begin{aligned}
\|(\overline{\boldsymbol{\lambda}}, \overline{\boldsymbol{\eta}})\|_{L^{\infty}((0, T), \boldsymbol{X})} \leq \|\left(\epsilon^{-1}\left(\overline{\boldsymbol{E}}-\boldsymbol{E}_{d}\right), \mu^{-1}\left(\overline{\boldsymbol{H}}-\boldsymbol{H}_{d}\right)\right) & \|_{L^{1}((0, T), \boldsymbol{X})} \\
& +\|(\overline{\boldsymbol{K}}, \overline{\boldsymbol{Q}})\|_{L^{\infty}((0, T), \boldsymbol{X})} .
\end{aligned}
$$

Let us now prove the estimate for $\|(\overline{\boldsymbol{K}}, \overline{\boldsymbol{Q}})\|_{L^{\infty}((0, T), \boldsymbol{X})}$. To this aim, we apply Hölder's inequality to (5.4) and obtain that

$$
\begin{array}{r}
\left\|\left(\overline{\boldsymbol{K}}^{\gamma}, \overline{\boldsymbol{Q}}^{\gamma}\right)\right\|_{L^{\infty}((0, T), \boldsymbol{X})} \leq 2 \max \left\{\underline{\epsilon}^{-1 / 2}, \underline{\mu}^{-1 / 2}\right\} T^{1 / 2}\left(\left\|\overline{\boldsymbol{E}}^{\gamma}-\boldsymbol{E}_{d}\right\|_{L^{2}\left((0, T), \boldsymbol{L}^{2}(\Omega)\right)}\right. \\
\left.+\left\|\overline{\boldsymbol{H}}^{\gamma}-\boldsymbol{H}_{d}\right\|_{L^{2}\left((0, T), \boldsymbol{L}^{2}(\Omega)\right)}\right) \quad \forall \gamma>0 .
\end{array}
$$

Copyright $\odot$ by SIAM. Unauthorized reproduction of this article is prohibited. 
Consequently, employing (4.42), it holds for every $\gamma>0$ that

$$
\begin{aligned}
& \left\|\left(\overline{\boldsymbol{K}}^{\gamma}, \overline{\boldsymbol{Q}}^{\gamma}\right)\right\|_{L^{\infty}((0, T), \boldsymbol{X})}^{2} \\
& \leq 8 \max \left\{\underline{\epsilon}^{-1}, \underline{\mu}^{-1}\right\} T\left(\left\|\overline{\boldsymbol{E}}^{\gamma}-\boldsymbol{E}_{d}\right\|_{L^{2}\left((0, T), \boldsymbol{L}^{2}(\Omega)\right)}^{2}+\left\|\overline{\boldsymbol{H}}^{\gamma}-\boldsymbol{H}_{d}\right\|_{L^{2}\left((0, T), \boldsymbol{L}^{2}(\Omega)\right)}^{2}\right) \\
& =16 \max \left\{\underline{\epsilon}^{-1}, \underline{\mu}^{-1}\right\} T\left(f_{\gamma}\left(\overline{\boldsymbol{u}}^{\gamma}\right)-\frac{\kappa}{2}\left\|\overline{\boldsymbol{u}}^{\gamma}\right\|_{H^{2}\left((0, T), \boldsymbol{L}^{2}(\Omega)\right)}^{2}-\frac{1}{2}\left\|\overline{\boldsymbol{u}}^{\gamma}-\overline{\boldsymbol{u}}\right\|_{H^{2}\left((0, T), \boldsymbol{L}^{2}(\Omega)\right)}^{2}\right) \\
& \leq 16 \max \left\{\underline{\epsilon}^{-1}, \underline{\mu}^{-1}\right\} T\left(f_{\gamma}(\overline{\boldsymbol{u}})-\frac{\kappa}{2}\left\|\overline{\boldsymbol{u}}^{\gamma}\right\|_{H^{2}\left((0, T), \boldsymbol{L}^{2}(\Omega)\right)}^{2}-\frac{1}{2}\left\|\overline{\boldsymbol{u}}^{\gamma}-\overline{\boldsymbol{u}}\right\|_{H^{2}\left((0, T), \boldsymbol{L}^{2}(\Omega)\right)}^{2}\right) \\
& =8 \max \left\{\underline{\epsilon}^{-1}, \underline{\mu}^{-1}\right\} T\left(\left\|S_{\gamma}(\overline{\boldsymbol{u}})-\left(\boldsymbol{E}_{d}, \boldsymbol{H}_{d}\right)\right\|_{L^{2}\left((0, T), \boldsymbol{L}^{2}(\Omega) \times \boldsymbol{L}^{2}(\Omega)\right)}^{2}+\kappa\|\overline{\boldsymbol{u}}\|_{H^{2}\left((0, T), \boldsymbol{L}^{2}(\Omega)\right)}^{2}\right) \\
& \left.\quad-\kappa\left\|\overline{\boldsymbol{u}}^{\gamma}\right\|_{H^{2}\left((0, T), \boldsymbol{L}^{2}(\Omega)\right)}^{2}-\left\|\overline{\boldsymbol{u}}^{\gamma}-\overline{\boldsymbol{u}}\right\|_{H^{2}\left((0, T), \boldsymbol{L}^{2}(\Omega)\right)}^{2}\right)
\end{aligned}
$$

where the last inequality follows from the fact that $\overline{\boldsymbol{u}}^{\gamma}$ is an optimal control of $\left(\mathrm{P}_{\gamma}^{\bar{u}}\right)$. Applying Lemma 4.10 and Theorem 4.11 to the above inequality, we deduce that

$$
\begin{aligned}
& \liminf _{\gamma \rightarrow \infty}\left\|\left(\overline{\boldsymbol{K}}^{\gamma}, \overline{\boldsymbol{Q}}^{\gamma}\right)\right\|_{L^{\infty}((0, T), \boldsymbol{X})}^{2} \leq \liminf _{\gamma \rightarrow \infty} I(\gamma) \leq \limsup _{\gamma \rightarrow \infty} I(\gamma) \\
& \leq 8 \max \left\{\underline{\epsilon}^{-1}, \underline{\mu}^{-1}\right\} T\left(\left\|\overline{\boldsymbol{E}}-\boldsymbol{E}_{d}\right\|_{L^{2}\left((0, T), \boldsymbol{L}^{2}(\Omega)\right)}^{2}+\left\|\overline{\boldsymbol{H}}-\boldsymbol{H}_{d}\right\|_{L^{2}\left((0, T), \boldsymbol{L}^{2}(\Omega)\right)}^{2}\right) .
\end{aligned}
$$

Thus, in view of (5.5), we come to the conclusion that

$$
\begin{aligned}
& \|(\overline{\boldsymbol{K}}, \overline{\boldsymbol{Q}})\|_{L^{\infty}((0, T), \boldsymbol{X})} \leq \liminf _{\gamma \rightarrow \infty}\left\|\left(\overline{\boldsymbol{K}}^{\gamma}, \overline{\boldsymbol{Q}}^{\gamma}\right)\right\|_{L^{\infty}((0, T), \boldsymbol{X})} \\
& \leq \sqrt{8 T} \max \left\{\underline{\epsilon}^{-1 / 2}, \underline{\mu}^{-1 / 2}\right\}\left(\left\|\overline{\boldsymbol{E}}-\boldsymbol{E}_{d}\right\|_{L^{2}\left((0, T), \boldsymbol{L}^{2}(\Omega)\right)}^{2}+\left\|\overline{\boldsymbol{H}}-\boldsymbol{H}_{d}\right\|_{L^{2}\left((0, T), \boldsymbol{L}^{2}(\Omega)\right)}^{2}\right)^{1 / 2} .
\end{aligned}
$$

Appendix. Let $(\boldsymbol{e}, \boldsymbol{h}) \in L^{2}((0, T), \boldsymbol{X})$ and $\boldsymbol{u}, \delta \boldsymbol{u} \in L^{2}\left((0, T), \boldsymbol{L}^{2}(\Omega)\right)$. We set $(\delta \boldsymbol{E}, \delta \boldsymbol{H})=S_{\gamma}^{\prime}(\boldsymbol{u}) \delta \boldsymbol{u}$ and $(\boldsymbol{E}, \boldsymbol{H})=G_{\gamma}(\boldsymbol{u})$. Employing the unitary structure of $\left\{\mathbb{T}_{t}\right\}_{t \in \mathbb{R}}$, we deduce that

$$
\begin{aligned}
& \left((\boldsymbol{e}, \boldsymbol{h}), S_{\gamma}^{\prime}(\boldsymbol{u}) \delta \boldsymbol{u}\right)_{L^{2}((0, T), \boldsymbol{X})}=((\boldsymbol{e}, \boldsymbol{h}),(\delta \boldsymbol{E}, \delta \boldsymbol{H}))_{L^{2}((0, T), \boldsymbol{X})} \\
& \underbrace{=}_{(4.35)} \int_{0}^{T}\left((\boldsymbol{e}, \boldsymbol{h})(t), \int_{0}^{t} \mathbb{T}_{t-s}\left(\epsilon^{-1}\left(\delta \boldsymbol{u}(s)-\nabla_{y} \varphi^{\gamma}(\cdot, \boldsymbol{E}(s)) \delta \boldsymbol{E}(s)\right), 0\right) d s\right)_{\boldsymbol{X}} d t \\
& =\int_{0}^{T} \int_{0}^{t}\left(\mathbb{T}_{t-s}^{*}(\boldsymbol{e}, \boldsymbol{h})(t),\left(\epsilon^{-1}\left(\delta \boldsymbol{u}(s)-\nabla_{y} \varphi^{\gamma}(\cdot, \boldsymbol{E}(s)) \delta \boldsymbol{E}(s)\right), 0\right)\right)_{\boldsymbol{X}} d s d t \\
& =\int_{0}^{T} \int_{0}^{t}\left(\mathbb{T}_{s-t}(\boldsymbol{e}, \boldsymbol{h})(t),\left(\epsilon^{-1}\left(\delta \boldsymbol{u}(s)-\nabla_{y} \varphi^{\gamma}(\cdot, \boldsymbol{E}(s)) \delta \boldsymbol{E}(s)\right), 0\right)\right)_{\boldsymbol{X}} d s d t \\
& =\int_{0}^{T}\left(\int_{t}^{T} \mathbb{T}_{t-s}(\boldsymbol{e}, \boldsymbol{h})(s) d s,\left(\epsilon^{-1}\left(\delta \boldsymbol{u}(t)-\nabla_{y} \varphi^{\gamma}(\cdot, \boldsymbol{E}(t)) \delta \boldsymbol{E}(t)\right), 0\right)\right)_{\boldsymbol{X}} d t \\
& \underbrace{=}_{(4.41 \mathrm{~b})} \int_{0}^{T}\left[\left((\boldsymbol{K}, \boldsymbol{Q})(t),\left(\epsilon^{-1}\left(\delta \boldsymbol{u}(t)-\nabla_{y} \varphi^{\gamma}(\cdot, \boldsymbol{E}(t)) \delta \boldsymbol{E}(t)\right), 0\right)\right)_{\boldsymbol{X}}\right. \\
& \left.\left.+\left(\int_{t}^{T} \mathbb{T}_{t-s}\left(\epsilon^{-1} \nabla_{y} \varphi^{\gamma}(\cdot, \boldsymbol{E}(s)) \boldsymbol{K}(s)\right), 0\right) d s,\left(\epsilon^{-1}\left(\delta \boldsymbol{u}(t)-\nabla_{y} \varphi^{\gamma}(\cdot, \boldsymbol{E}(t)) \delta \boldsymbol{E}(t)\right), 0\right)\right)_{\boldsymbol{X}}\right] d t
\end{aligned}
$$

Copyright (? by SIAM. Unauthorized reproduction of this article is prohibited. 


$$
\begin{aligned}
& =\int_{0}^{T}\left[(\boldsymbol{K}(t), \delta \boldsymbol{u}(t))_{\boldsymbol{L}^{2}(\Omega)}-\left(\boldsymbol{K}(t), \nabla_{y} \varphi^{\gamma}(\cdot, \boldsymbol{E}(t)) \delta \boldsymbol{E}(t)\right)_{\boldsymbol{L}^{2}(\Omega)}\right. \\
& \left.\left.+\left(\left(\epsilon^{-1} \nabla_{y} \varphi^{\gamma}(\cdot, \boldsymbol{E}(t)) \boldsymbol{K}(t)\right), 0\right), \int_{0}^{t} \mathbb{T}_{t-s}\left(\epsilon^{-1}\left(\delta \boldsymbol{u}(s)-\nabla_{y} \varphi^{\gamma}(\cdot, \boldsymbol{E}(s)) \delta \boldsymbol{E}(s)\right), 0\right) d s\right)_{\boldsymbol{X}}\right] d t \\
& \underbrace{=}_{(4.35)} \int_{0}^{T}\left[(\boldsymbol{K}(t), \delta \boldsymbol{u}(t))_{\boldsymbol{L}^{2}(\Omega)}-\left(\boldsymbol{K}(t), \nabla_{y} \varphi^{\gamma}(\cdot, \boldsymbol{E}(t)) \delta \boldsymbol{E}(t)\right)_{\boldsymbol{L}^{2}(\Omega)}\right. \\
& \left.\left.\quad+\left(\left(\epsilon^{-1} \nabla_{y} \varphi^{\gamma}(\cdot, \boldsymbol{E}(t)) \boldsymbol{K}(t)\right), 0\right),(\delta \boldsymbol{E}, \delta \boldsymbol{H})(t)\right)_{\boldsymbol{X}}\right] d t \\
& =\int_{0}^{T}\left[(\boldsymbol{K}(t), \delta \boldsymbol{u}(t))_{\boldsymbol{L}^{2}(\Omega)}-\left(\boldsymbol{K}(t), \nabla_{y} \varphi^{\gamma}(\cdot, \boldsymbol{E}(t)) \delta \boldsymbol{E}(t)\right)_{\boldsymbol{L}^{2}(\Omega)}\right. \\
& \left.\quad+\left(\nabla_{y} \varphi^{\gamma}(\cdot, \boldsymbol{E}(t)) \boldsymbol{K}(t), \delta \boldsymbol{E}(t)\right)_{\boldsymbol{L}^{2}(\Omega)}\right] d t \\
& =\int_{0}^{T}(\boldsymbol{K}(t), \delta \boldsymbol{u}(t))_{\boldsymbol{L}^{2}(\Omega)} d t=(\boldsymbol{K}, \delta \boldsymbol{u})_{L^{2}\left((0, T), \boldsymbol{L}^{2}(\Omega)\right) .}
\end{aligned}
$$

In conclusion, (4.41) is valid.

\section{REFERENCES}

[1] A. Alonso And A. VAlli, Eddy Current Approximation of Maxwell Equations: Theory, Algorithms and Applications, Springer, New York, 2010.

[2] C. Amrouche, C. Bernardi, M. Dauge, and V. Girault, Vector potentials in threedimensional non-smooth domains, Math. Methods Appl. Sci., 21 (1998), pp. 823-864.

[3] J. M. BALL, Strongly continuous semigroups, weak solutions, and the variation of constants formula, Proc. Amer. Math. Soc., 64 (1977), pp. 370-373.

[4] V. Barbu, Optimal Control of Variational Inequalities, Pitman, Boston, MA, 1984.

[5] J. W. Barrett And L. Prigozhin, A quasi-variational inequality problem in superconductivity, Math. Models Methods Appl. Sci., 20 (2010), pp. 679-706.

[6] C. P. Bean, Magnetization of hard superconductors, Phys. Rev. Lett., 8 (1962), pp. 250-253.

[7] C. P. Bean, Magnetization of high-field superconductors, Rev. Mod. Phys., 36 (1964), pp. 31-39.

[8] V Bommer and I. Yousept, Optimal control of the full time-dependent Maxwell equations, ESAIM Math. Model. Numer. Anal., 50 (2016), pp. 237-261.

[9] C. M. Elliott and Y. Kashima, A finite-element analysis of critical-state models for type-II superconductivity in 3D, IMA J. Numer. Anal., 27 (2007), pp. 293-331.

[10] K.-J. Engel And R. NAGEL, One-Parameter Semigroups for Linear Evolution Equations, Grad. Texts in Math. 194, Springer-Verlag, New York, 2000.

[11] R. H. W. Hoppe And I. Yousept, Adaptive edge element approximation of H(curl)-elliptic optimal control problems with control constraints, BIT, 55 (2015), pp. 255-277.

[12] K. Ito And K. Kunisch, Optimal control of parabolic variational inequalities, J. Math. Pures Appl. (9), 93 (2010), pp. 329-360.

[13] F. Jochmann, On a first-order hyperbolic system including Bean's model for superconductors with displacement current, J. Differential Equations, 246 (2009), pp. 2151-2191.

[14] F. Jochmann, A variational inequality in Bean's model for superconductors with displacement current, Discrete Contin. Dyn. Syst., 25 (2009), pp. 545-565.

[15] S. Nicaise, S. Stingelin, and F. Tröltzsch, On two optimal control problems for magnetic fields, Comput. Methods Appl. Math., 14 (2014), pp. 555-573.

[16] A. PAZY, Semigroups of Linear Operators and Applications to Partial Differential Equations, Springer-Verlag, New York, 1983.

[17] L. Prigozhin, The Bean model in superconductivity: Variational formulation and numerical solution, J. Comput. Phys., 129 (1996), pp. 190-200.

[18] L. Prigozhin, On the Bean critical-state model in superconductivity, European J. Appl. Math., 7 (1996), pp. 237-247.

Copyright (c) by SIAM. Unauthorized reproduction of this article is prohibited. 
[19] J. F. Rodrigues And L. SAntos, A parabolic quasi-variational inequality arising in a superconductivity model, Ann. Scuola Norm. Sup. Pisa Cl. Sci. (4), 29 (2000), pp. 153-169.

[20] R. E. Showalter, Monotone Operators in Banach Space and Nonlinear Partial Differential Equations, Math. Surveys Monographs 49, AMS, Providence, RI, 1997.

[21] F. Tröltzsch, Optimal Control of Partial Differential Equations, Grad. Stud. Math. 112, AMS, Providence, RI, 2010.

[22] F. Tröltzsch And I. Yousept, PDE-constrained optimization of time-dependent $3 D$ electromagnetic induction heating by alternating voltages, ESAIM Math. Model. Numer. Anal., 46 (2012), pp. 709-729.

[23] I. Yousept, Finite element analysis of an optimal control problem in the coefficients of timeharmonic eddy current equations, J. Optim. Theory Appl., 154 (2012), pp. 879-903.

[24] I. Yousept, Optimal control of Maxwell's equations with regularized state constraints, Comput. Optim. Appl., 52 (2012), pp. 559-581.

[25] I. Yousept, Optimal control of quasilinear $\boldsymbol{H}$ (curl)-elliptic partial differential equations in magnetostatic field problems, SIAM J. Control Optim., 51 (2013), pp. 3624-3651.

Copyright (? by SIAM. Unauthorized reproduction of this article is prohibited. 